\title{
Mean Field Derivation of DNLS from the Bose-Hubbard Model
}

\author{
E. Picari, A. Ponno and L. Zanelli
}

\begin{abstract}
We prove that the flow of the discrete nonlinear Schrödinger equation (DNLS) is the mean field limit of the quantum dynamics of the Bose-Hubbard model for $\mathrm{N}$ interacting particles. In particular, we show that the Wick symbol of the annihilation operators evolved in the Heisenberg picture converges, as $N$ becomes large, to the solution of the DNLS. A quantitative $L^{p}$-estimate, for any $p \geq 1$, is obtained with a linear dependence on time due to a Gaussian measure on initial data coherent states.
\end{abstract}

\section{Introduction}

The DNLS equation considered in the present paper is the following

$$
i \frac{d}{d t} u_{k}(t)=E u_{k}(t)+J\left(u_{k+1}(t)+u_{k-1}(t)\right)+U\left|u_{k}(t)\right|^{2} u_{k}(t),
$$

with initial data $u_{k}(0):=\omega_{k} \in \mathbb{C}, 1 \leq k \leq L$ and boundary conditions $u_{1}(t)=$ $u_{L+1}(t), \omega_{L+1}=\omega_{1}$ (see [2]); we denote its flow by $u(t, \omega):=\left(u_{1}, \ldots u_{L}\right)(t, \omega)$. The Hamilton operator of the Bose-Hubbard model, which describes cold atoms in a deep one-dimensional optical lattice (see $[12,19,40]$ ), is

$$
\widehat{H}:=\sum_{1 \leq j \leq L}\left[E \hat{b}_{j}^{\dagger} \hat{b}_{j}+J\left(\hat{b}_{j+1}^{\dagger} \hat{b}_{j}+\hat{b}_{j}^{\dagger} \hat{b}_{j+1}\right)+\frac{U}{2 N} \hat{b}_{j}^{\dagger} \hat{b}_{j}^{\dagger} \hat{b}_{j} \hat{b}_{j}\right],
$$

depending on the usual bosonic operators satisfying $\left[\hat{b}_{k}, \hat{b}_{\mu}^{\dagger}\right]=\delta_{k \mu} \operatorname{Id},\left[\hat{b}_{k}, \hat{b}_{\mu}\right]=$ 0 and defined on the Fock-Bargmann space $\mathcal{F}_{B}\left(\mathbb{C}^{L}\right)$, see $[15,23]$ and Sect. 3.1. In (2), $E, J$ and $U$ are real parameters, whereas $N$ is the expected number of bosons in the lattice.

The aim of the present paper is to show that the quantum expectation of $\hat{b}_{k} / \sqrt{N}$ is close to $u_{k}(t, \omega)$ in a suitable $L^{p}$-measure sense when $N$ is large, which is precisely what we mean by the mean field limit of (2). This is achieved 
thanks to an explicit estimate in terms of the parameters of the model, global in time.

The literature on the mean field derivation of the nonlinear Schrödinger equation (NLS), the Hartree equation, and more in general about the study of many-body quantum-mechanical systems, is quite rich (see Sect. 2). However, it seems that a direct mean field derivation of the DNLS (1) together with quantitative, explicit estimates is missing. In some works (see for example $[1,33,37]$ and references therein) the DNLS is obtained directly from the NLS equation, in the framework of the tight-binding approximation. However, combining these two kinds of results, a growth exponential in time of the mean field estimate for DNLS follows (essentially due to the Grönwall lemma). In the present paper, a growth linear in time of the mean field estimate is provided. Our first step to deal with the mean field asymptotics is to consider the rescaled operators

$$
\hat{a}_{k}:=\frac{\hat{b}_{k}}{\sqrt{N}}, \quad \hat{a}_{k}^{\dagger}:=\frac{\hat{b}_{k}^{\dagger}}{\sqrt{N}}, \quad\left[\hat{a}_{k}, \hat{a}_{\mu}^{\dagger}\right]=\frac{\delta_{k \mu}}{N} \mathrm{Id},
$$

and the Heisenberg equation $i \frac{d}{d t} \hat{a}_{k}(t)=\left[\widehat{H}, \hat{a}_{k}(t)\right]$ that reads

$$
i \frac{d}{d t} \hat{a}_{k}(t)=E \hat{a}_{k}(t)+J\left(\hat{a}_{k+1}(t)+\hat{a}_{k-1}(t)\right)+U \hat{a}_{k}^{\dagger}(t) \hat{a}_{k}(t) \hat{a}_{k}(t),
$$

where $\hat{a}_{k}(0):=\hat{a}_{k}$ and $1 \leq k \leq L$. Notice that Eq. (4) is clearly the operator counterpart of (1). We now rewrite Eq. (4) in terms of the Wick symbols (see Sect. 3.1) of the operators $\hat{a}_{k}(t)$ and $\widehat{H}$. Let $\phi_{\omega}(\bar{z}):=e^{\omega \cdot \bar{z}-\frac{1}{2}|\omega|^{2}}$ be the normalized coherent states in $\mathcal{F}_{B}\left(\mathbb{C}^{L}\right)$ and recall that $\hat{a}_{k} \phi_{\sqrt{N} \omega}=\omega_{k} \phi_{\sqrt{N} \omega}$. Define the symbols

$$
\begin{aligned}
\rho_{k}(t, \bar{\omega}, \omega) & :=\left\langle\phi_{\sqrt{N} \omega}, \hat{a}_{k}(t) \phi_{\sqrt{N} \omega}\right\rangle \\
H_{N}(\bar{\omega}, \omega) & :=\left\langle\phi_{\sqrt{N} \omega}, \widehat{H} \phi_{\sqrt{N} \omega}\right\rangle=N \mathcal{H}(\bar{\omega}, \omega) \\
& =N \sum_{1 \leq j \leq L}\left[E_{j}\left|\omega_{j}\right|^{2}+J\left(\bar{\omega}_{j+1} \omega_{j}+\bar{\omega}_{j}, \omega_{j+1}\right)+\frac{U}{2}\left|\omega_{j}\right|^{4}\right] .
\end{aligned}
$$

Then, by the Wick bracket (see $[9,15])$ we get the equation

$$
\frac{i}{N} \frac{d}{d t} \rho_{k}=\left\{\rho_{k}, \mathcal{H}\right\}_{\text {Wick }}
$$

with initial data $\rho_{k}(0, \omega, \bar{\omega})=\omega_{k}$. We recall that, as an asymptotic series,

$$
\begin{aligned}
\left\{\rho_{k}, \mathcal{H}\right\}_{\text {Wick }} & :=\rho_{k} \star_{\text {Wick }} \mathcal{H}-\mathcal{H} \star_{\text {Wick }} \rho_{k} \\
& \simeq \sum_{r=1}^{\infty} \frac{1}{r !}\left(\frac{1}{N}\right)^{r}\left(\frac{\partial^{r} \rho_{k}}{\partial \omega^{r}} \frac{\partial^{r} \mathcal{H}}{\partial \bar{\omega}^{r}}-\frac{\partial^{r} \mathcal{H}}{\partial \omega^{r}} \frac{\partial^{r} \rho_{k}}{\partial \bar{\omega}^{r}}\right),
\end{aligned}
$$

where $\star_{\text {Wick }}$ denotes the usual Wick-star product; see "Appendix" for details. In view of (8)-(9), we recognize the role of $1 / N$ as a semiclassical parameter. Since $\mathcal{H}$ is a second-order polynomial of complex variables $(\omega, \bar{\omega})$, it follows 
that here the Wick bracket is a finite $\operatorname{sum}\{\cdot, \mathcal{H}\}_{\text {Wick }}=\mathcal{L}_{1}+\mathcal{L}_{2}$ where

$$
\mathcal{L}_{1}:=\frac{1}{N}\left(\frac{\partial \mathcal{H}}{\partial \bar{\omega}} \frac{\partial}{\partial \omega}-\frac{\partial \mathcal{H}}{\partial \omega} \frac{\partial}{\partial \bar{\omega}}\right), \quad \mathcal{L}_{2}:=\frac{1}{2 N^{2}}\left(\frac{\partial^{2} \mathcal{H}}{\partial \bar{\omega}^{2}} \frac{\partial^{2}}{\partial \omega^{2}}-\frac{\partial^{2} \mathcal{H}}{\partial \omega^{2}} \frac{\partial^{2}}{\partial \bar{\omega}^{2}}\right) .
$$

Notice that $\mathcal{L}_{1}$ is the Poisson bracket in the variables $(\bar{\omega}, \omega)$. Thus, it is easily seen that the DNLS (1) exactly reads

$$
\frac{i}{N} \frac{d}{d t} u=\mathcal{L}_{1}(u)
$$

By denoting $\Delta:=\left\{(\bar{\omega}, \omega) \mid \omega \in \mathbb{C}^{L}\right\} \subset \mathbb{C}^{2 L}$, and $\left(\bar{\Phi}_{t}, \Phi_{t}\right): \Delta \subset \mathbb{C}^{2 L} \rightarrow \mathbb{C}^{2 L}$ the flow of $\dot{\gamma}=i\left(\partial_{\omega} \mathcal{H}(\gamma),-\partial_{\bar{\omega}} \mathcal{H}(\gamma)\right)$, it follows that

$$
u(t, \omega)=\Phi_{t}(\bar{\omega}, \omega) .
$$

The equality (12), together with Eq. (8), tells us that $\rho_{k}-u_{k}$ is a kind of semiclassical perturbation term, and thus we expect $\rho_{k}-u_{k} \rightarrow 0$ as $N \rightarrow+\infty$. Indeed, we will prove such a result with respect to an $L^{p}\left(\mu_{N}\right)$-norm, where $p \geq 1$ and $\mu_{N}$ is a suitable Gaussian measure, invariant under the DNLS flow. With respect to this target, recall that the total number operator defined as $\widehat{N}:=\sum_{k=1}^{L} \hat{b}_{k}^{\dagger} \hat{b}_{k}=N \sum_{k=1}^{L} \hat{a}_{k}^{\dagger} \hat{a}_{k}$ fulfills $[\widehat{H}, \widehat{N}]=0$ and hence

$$
\left\langle\phi_{\sqrt{N} \omega}, \widehat{N} \phi_{\sqrt{N} \omega}\right\rangle=N|\omega|^{2}
$$

is conserved by the quantum flow, i.e.,

$$
\left\{\mathcal{H},|\omega|^{2}\right\}_{\text {Wick }}=0 \text {. }
$$

Moreover, the well-known $\ell^{2}$-conservation law for the DNLS can be rewritten as

$$
\mathcal{L}_{1}\left(|\omega|^{2}\right)=0
$$

Both these two important properties will be used in the proof of Theorem 1, and for this reason we define the invariant Gaussian probability measure

$$
d \mu_{N}(\bar{\omega}, \omega):=c_{N, L} e^{-N|\omega|^{2}} d \omega \wedge d \bar{\omega},
$$

where $\omega=x+i y, d \omega \wedge d \bar{\omega}:=\pi^{-L} d x d y$ and $c_{N, L}:=N^{L}$ is the normalization constant. This measure is linked (see Proposition 1) to a weighted trace formula involving Wick operators that will be an important tool to our approach.

We are now ready to state the main result of the paper.

Theorem 1. Let $u(t, \omega)$ be the flow of the DNLS Eq. (1) and let $\rho_{k}(t, \omega)$ be the solution of (8) for $1 \leq k \leq L$. Then, $\forall p \geq 1$ we have $u_{k}, \rho_{k} \in L^{p}\left(\mu_{N}\right)$ and

$$
\left\|\rho_{k}(t)-u_{k}(t)\right\|_{L^{p}\left(\mu_{N}\right)} \leq A_{p} \frac{L}{N} \frac{U t}{\sqrt{N}}, \quad \forall t \geq 0
$$

with the constant $A_{p}=B_{2^{p}}, B_{\tau}:=\left(C_{1, \tau} \cdot C_{2, \tau}\right)^{\frac{1}{\tau}}$, where

$$
C_{1, \tau}:=\left(\sum_{\alpha_{1}+\alpha_{2}+\alpha_{3}=4 \tau}\left(\begin{array}{c}
4 \tau \\
\alpha_{1} \alpha_{2} \alpha_{3}
\end{array}\right) 3^{\alpha_{1}} 2^{2 \alpha_{2}+\frac{1}{2} \alpha_{3}} \Gamma\left(2 \alpha_{1}+\frac{3}{2} \alpha_{2}+\alpha_{3}+1\right)\right)^{\frac{1}{4}}
$$




$$
C_{2, \tau}:=\left(\sum_{\alpha=1}^{2 \tau}\left(\begin{array}{c}
2 \tau \\
\alpha
\end{array}\right) \sum_{\beta=1}^{\alpha} S(\alpha, \beta) \beta !\right)^{\frac{1}{4}} .
$$

Here, $\Gamma$ and $S(\alpha, \beta)$ denote the Gamma function and the Stirling numbers of second kind, respectively.

We notice that (17) can be written with the condition $L / N \rightarrow 0$ as $N \rightarrow+\infty$, this means that the number of particles can be supposed to be large with respect to the number of $L$ "sites" of the Bose-Hubbard model, which is the regime considered in some experiments, see for example [40].

We also stress that the $L^{p}$-norm used above allows to discuss, in the measure sense, the pointwise estimate for $\left|\rho_{k}-u_{k}\right|(t, \bar{\omega}, \omega)$. Indeed, we have the following

Corollary 1. Fix a parameter $0<\epsilon<\frac{1}{2}$ and define the set

$$
\Omega_{k}:=\left\{(\bar{\omega}, \omega)|\quad| \rho_{k}-u_{k} \mid(t, \bar{\omega}, \omega)>A_{p} \frac{L}{N} \frac{U t}{N^{\epsilon}}, \quad \forall t \geq 0\right\} .
$$

Then, for any $1 \leq k \leq L$

$$
\mu_{N}\left(\Omega_{k}\right) \leq N^{-p \cdot\left(\frac{1}{2}-\epsilon\right)}, \quad \forall p \geq 1 \quad \forall N \geq 1 .
$$

Notice that $p \mapsto A_{p}$ is an increasing function, whence inequality (21) provides, when $N$ is fixed and $p$ is large, a measure of the region where $\left|\rho_{k}-u_{k}\right|$ is large.

On the other hand, in the case of a fixed $p \geq 1$ and large values of $N$ we have a vanishing measure of the region where $\left|\rho_{k}-u_{k}\right|$ is super-linear in time. We underline an important consequence of this observation. Indeed this means that, from the viewpoint of the Gaussian measure, if this superlinear (in time) mean field estimate is sharp then it is associated with a set of coherent states which is negligible as $N \rightarrow+\infty$ with the rate shown in (21). Of course, any exponential (in time) upper bound gives rise to the same conclusion. An interesting open problem is to show that the same feature holds for more general quantum dynamics than the one associated with our manybody operator (2).

Our paper deals with the Bose-Hubbard model, a simpler setting with respect to that of quantum field theory. However, the explicit estimate in terms of the parameters of the model and its linear dependence on time in (17) seem to be a novel and promising result with respect to other kind of mean field estimates on the NLS equation.

Furthermore, we stress that Theorem 1 can be seen as an Egorov-type result, written to the first order and with respect to the $L^{p}$-norm, for Wick symbols. With respect to this observation, we recall Proposition 5.1 in [16] where it is proved the convergence, as $\hbar \rightarrow 0$, of the Wick symbol of an evolved quantum observable toward the Weyl symbol composed with the Hamiltonian flow. In this result, the well-known bound of the Ehrenfest time $|t|<T_{\hbar}$ is shown. We also recall Proposition 5.10 and Theorem 5.6 in [5] where, in the framework of evolved Wick operators on the Fock space and with a quantum dynamics much more general than our, it is proved the convergence toward the 
solution of the Hartree equation as $\frac{1}{N} \rightarrow 0$, but the estimate on the remainder in Theorem 5.6 is again local in time. In our main result, we avoid locality in time by making use of the $L^{p}\left(\mu_{N}\right)$-norm (the meaning and the properties of the measure $\mu_{N}$ are clarified in Proposition 1 and 2).

In Sect. 7.2 of [26], the authors discuss, thanks to the Wick quantization for a class of symbols, how the many-body quantum mechanics of bosons can be viewed as a deformation quantization of the Hartree theory. We stress that our paper also makes use of Wick operators, but deals with a different class of quantum dynamics and another way to get the derivation of the mean field dynamics, which is here a discrete NLS.

To conclude, we stress the absence in (17) of the parameters $E$ and $J$ involved in the quadratic part of the operator $\widehat{H}$ in (2), in agreement with a well-known elementary result: any quantum expectation of the Heisenberg equations of a linear system (quadratic Hamiltonian) yields the classical equations of motion. Thus, the distance between the Wick symbol $\rho_{k}$ solving Eq. (8) and the $k$-th component $u_{k}$ of the flow for Eq. (11) is ruled only by nonlinearity, namely by the parameter $U$. As a consequence, Theorem 1 holds for Hamilton operators $\widehat{H}$ with a completely general quadratic part. This is not the primary target of the present work, but we observe here that a more general setting of $\widehat{H}$ ensures a larger set of invariant measures for the DNLS flow and whence an interesting open problem is to study the link between this kind of mean field estimates and the possible various invariant measures.

We also remark that the equation in (1) with general quadratic part, that usually describes particles in one-dimensional periodic lattice, can be used also to modelize two- and three-dimensional lattices with different topologies (see for example [22] and references therein).

The paper is organized as follows. In Sect. 2, we shortly comment on the most influential results in the literature on the subject, to finally stress the main innovations characterizing our work. Sect. 3 is devoted to the proofs of Theorem 1 and Corollary 1 stated in the Introduction; the proof is divided into various technical steps. Sect. 3.1 is "Appendix" consisting of three subsections.

\section{Synopsis of the Literature and Motivations of the Work}

We here provide a commented list of some papers involving NLS, Hartree equation, and more in general the study of many-body quantum-mechanical systems in mean field and semiclassical limit, relevant and somehow connected to our work.

A first reference work in the field, is the review [39], where the author discusses a variety of classical as well as quantum models for which kinetic equations can be derived rigorously, and where the probabilistic nature of the problem is emphasized.

A second reference paper, of particular interest to our work for its use of coherent states, is [29], where Hepp shows that in the many-body framework the classical limit of the expectation values of products of Weyl operators, 
translated in time by the quantum dynamics and taken on coherent states centered in $x$-space and $p$-space are shown to become the exponentials of coordinate functions of the classical orbit in phase space. The Hepp results have been extended in [27]. For a recent review and discussion of Hepp's method, we also address the reader to Sect. 10.4.2 of [21].

The convergence of the $N$-particle Schrödinger dynamics of bosons toward the Hartree dynamics is proved in the mean field limit in [24]. The authors work in the Heisenberg picture (as in our paper) with a class of bounded operators, whereas we consider annihilation operators, that are unbounded, and another type of convergence.

A rigorous derivation of the cubic NLS in dimension one is shown in [4]. An approach for deriving higher-order corrections to the mean field limit for the quantum systems is provided in [11], a simple and effective method is given in $[35]$.

A reference role in the literature is played by those recent works dealing with the rigorous version of the Bogoliubov theory of superfluids; see, e.g., the review [38]. The Gross-Pitaevskii equation is rigorously deduced, for example, in [14], whereas the fluctuations around it are studied in $[13,17]$. The convergence to a limiting Hartree dynamics is instead studied in $[6,36]$, whereas in $[7,10]$ and the Hartree-Fock-Bogoliubov and the Bogoliubov-de Gennes equations are derived by the method of the quasi-free reduction.

Further results are, for example, a derivation of the 1D focusing cubic NLS obtained in [20], where the difficulties due to the attractive interaction are discussed and new energy estimates are shown, and a mean field derivation of the defocusing 2D cubic NLS is provided in [28]; the mean field dynamics of a mixture of bosons is treated in [32].

The literature on the subject is actually huge, and the above description represents just a short summary of it. However, with the respect to the existing framework of methods and results, our contribution here is characterized by a certain number of aspects deserving a short discussion.

1. We consider the Bose-Hubbard lattice model (2). This is certainly a context much simpler than the quantum field theory of a boson gas generally considered in the literature quoted above. However, its interest is motivated by the modern experiments on many-body effects in optical lattices, where the lattice models are the basic tool for the theoretical interpretation of the results $[12,19]$.

2. The privileged physical quantity considered here is the coherent expectation of the local annihilation operator, which is shown to satisfy the DNLS equation within a certain approximation limit. As specified below, we would be able to do the same with any observable, say any polynomial of the Dirac operators. The choice of the annihilation operator, besides its simplicity, is quite natural if, along an Ehrenfest-like line of thought, one wants to compare the quantum expectation of the Heisenberg equation of a certain operator with the "classical" scalar equation obtained by 
replacing the operator with its quantum expectation in the Heisenberg equation itself, as heuristically done in physics.

3. A clear innovation of our approach consists in distributing the initial data (i.e., coherent states) according to an invariant probability measure, which then calls naturally for the use of the $L^{2}$ norm (which is then generalized to $L^{p}$ ), as typical and meaningful in statistical mechanics, the right framework for this kind of problems; for the experimental relevance of coherent states see $[12,19]$. However, in certain applications, e.g., quantum computing, special initial conditions, and thus point-wise estimates, may play the relevant role. Indeed, we refer for example to the quantum walks in the Bose-Hubbard model, that are unitary processes describing the evolution of initially localized wave functions on a lattice potential, see [30].

Concerning the choice of the measure, we take the Gaussian one, inherited by the quantum trace measure with density $e^{-\lambda \widehat{N}}, \lambda$ being a suitable parameter. Of course, in a statistical mechanical framework, one would like to work with the Gibbs-Von Neumann density, namely $e^{-\beta \widehat{H}}$, $\beta$ being the inverse temperature. The latter point makes part of a work in progress. Here, we only stress that the Hamiltonian (2) reads $\widehat{H}=$ $E \widehat{N}+\cdots$, the dots denoting the other two terms, $\widehat{N}$ commuting with both of them. As will be further discussed below, and expected from the tight-binding assumptions made to deduce the Bose-Hubbard model, the first term $E \widehat{N}$ is the leading one with respect to the other two. In a sense, we are thus considering an invariant measure that is approximately connected to the Gibbs one.

4. The joint use of an invariant measure on the initial coherent states and of the Wick formalism allows us to bound distance between the symbol of an observable at time $t$ and the classical evolution of its initial symbol by a constant growing linearly with $t$. The linear dependence on time is not a particular feature of the Gaussian measure, the latter being instead quite convenient in order to get an explicit estimate of the overall constant multiplying time. Within the framework of results in measure on coherent states, the linear growth in time of our bound represents an interesting news, since most of time dependencies obtained in the literature up to now are typically exponential, which is an unavoidable consequence of the Grönwall lemma.

\section{Results}

In this section, we provide the proof of the main theorem we have stated in the Introduction. To such a purpose, we will need some preliminary lemmas and propositions. Among them, Lemmas 1 and 2 are just quoted and used, their statements and proofs being reported at the end of the section.

The following result provides a weighted trace formula for Wick operators, involving the positive definite operator $e^{-\lambda \widehat{N}}$ with $\lambda>0$. In order to make a 
link with the Gaussian measure $\mu_{N}$ given in (16), we have to write a bijective relation between the parameters $\lambda$ and $N$. This result will be useful for the subsequent result on expectation values of Wick operators under quantum dynamics.

Proposition 1. Let $\mu_{N}$ be as in (16). Let $\mathrm{Op}_{\mathrm{W}}(g)$ be a Wick operator on $\mathcal{F}_{B}\left(\mathbb{C}^{L}\right)$,

$$
\mathrm{Op}_{\mathrm{W}}(g)(\psi)(\bar{z}):=\int g(\bar{z}, \omega) \psi(\bar{\omega}) e^{-|\omega|^{2}+\omega \cdot \bar{z}} d \omega \wedge d \bar{\omega}, \quad \psi \in \mathcal{F}_{B}\left(\mathbb{C}^{L}\right),
$$

such that $g \in L^{1}\left(\mu_{N}\right)$. Let $\widehat{N}:=\sum_{k=1}^{L} \hat{b}_{k}^{\dagger} \hat{b}_{k}$. Then,

$$
\operatorname{Tr}\left(\frac{e^{-\lambda \widehat{N}}}{\gamma_{\lambda}} \mathrm{Op}_{\mathrm{W}}(g)\right)=\int g d \mu_{N}
$$

where $\gamma_{\lambda}:=\operatorname{Tr}\left(e^{-\lambda \widehat{N}}\right)$ and $e^{\lambda}=N+1$.

Proof. We begin by the equality

$$
\operatorname{Tr}\left(\frac{e^{-\lambda \widehat{N}}}{\gamma_{\lambda}} \mathrm{Op}_{\mathrm{W}}(g)\right)=\int \sigma\left(\frac{e^{-\lambda \widehat{N}}}{\gamma_{\lambda}} \mathrm{Op}_{\mathrm{W}}(g)\right)(\bar{\omega}, \omega) d \omega \wedge d \bar{\omega}
$$

and notice that the Wick symbol of $e^{-\lambda \widehat{N}}$ reads

$$
\sigma\left(e^{-\lambda \widehat{N}}\right)(\bar{\omega}, \omega)=e^{-\mu|\omega|^{2}}, \quad \mu:=1-e^{-\lambda} .
$$

Equality (25) allows to write the constant

$$
\gamma_{\lambda}=\operatorname{Tr}\left(e^{-\lambda \widehat{N}}\right)=\int \sigma\left(e^{-\lambda \widehat{N}}\right)(\bar{\omega}, \omega) d \omega \wedge d \bar{\omega}=\left(\frac{1}{\mu}\right)^{L} .
$$

Thanks to the Wick-^ product, (24) can be rewritten as

$$
\frac{1}{\gamma_{\lambda}} \int e^{-\mu|\omega|^{2}} \star_{\text {Wick }} g(\bar{\omega}, \omega) d \omega \wedge d \bar{\omega} \text {. }
$$

We also remind formula (2.38) in [15] that provides a link between Wick and anti-Wick symbols

$$
e^{-\mu|\omega|^{2}}=e^{\Delta_{\bar{\omega} \omega}} \sigma_{\mathrm{AW}}\left(e^{-\lambda \widehat{N}}\right)=\int e^{-(z-\omega)(\bar{z}-\bar{\omega})} \sigma_{\mathrm{AW}}(z, \bar{z}) d z \wedge d \bar{z},
$$

where $\Delta_{\bar{\omega} \omega}:=\sum_{k=1}^{L} \frac{\partial^{2}}{\partial \bar{\omega}_{k} \partial \omega_{k}}$, and recall that Wick and anti-Wick symbols of $e^{-\lambda \widehat{N}}$ are unique. Now write explicitly the Wick-^ product and integrate by parts the integral in (27). This gives (formally)

$$
\frac{1}{\gamma_{\lambda}} \int\left(e^{-\Delta_{\bar{\omega} \omega}} e^{-\mu|\omega|^{2}}\right) g(\bar{\omega}, \omega) d \omega \wedge d \bar{\omega}
$$

Recall that

$$
d \mu_{N}(\bar{\omega}, \omega):=c_{N, L} e^{-N|\omega|^{2}} d \omega \wedge d \bar{\omega}
$$

where $c_{N, L}:=N^{L}$. Our target is thus to prove the well-defined equation

$$
\gamma_{\lambda}^{-1} e^{-\mu|\omega|^{2}}=c_{N, L} e^{\Delta_{\bar{\omega} \omega}} e^{-N|\omega|^{2}}
$$


namely

$$
\begin{aligned}
e^{-\mu|\omega|^{2}} & =\gamma_{\lambda} c_{N, L} \int e^{-(z-\omega)(\bar{z}-\bar{\omega})} e^{-N|z|^{2}} d z \wedge d \bar{z} \\
& =\mu^{-L} N^{L} e^{-\frac{N}{N+1}|\omega|^{2}} \int e^{-(N+1)|z|^{2}} d z \wedge d \bar{z} \\
& =\mu^{-L}\left(\frac{N}{N+1}\right)^{L} e^{-\frac{N}{N+1}|\omega|^{2}}
\end{aligned}
$$

which is solved by $\mu=N /(N+1)$, and since $\mu=1-e^{-\lambda}$ we recover

$$
e^{\lambda}=N+1 \text {. }
$$

Remark 1. We now recall that $\hat{b}_{k}^{\dagger} \hat{b}_{\mu}=\mathrm{Op}_{\mathrm{W}}(g)$ when $g=\bar{\omega}_{k} \omega_{\mu}$, (see Sect. 3.1). For these Wick operators, Proposition 1 reads

$$
\operatorname{Tr}\left(\frac{e^{-\lambda \widehat{N}}}{\gamma_{\lambda}} \hat{b}_{k}^{\dagger} \hat{b}_{\mu}\right)=\int \bar{\omega}_{k} \omega_{\mu} d \mu_{N}
$$

Since $\left\{\frac{\bar{z}^{\alpha}}{\sqrt{\alpha !}}, \alpha \in \mathbb{Z}_{+}^{n}\right\}$ is an orthonormal set in the Fock-Bargmann space (see [15]), an easy computation shows that

$$
\operatorname{Tr}\left(\frac{e^{-\lambda \widehat{N}}}{\gamma_{\lambda}} \hat{b}_{k}^{\dagger} \hat{b}_{\mu}\right)=\frac{\delta_{k \mu}}{N}=\frac{\delta_{k \mu}}{e^{\lambda}-1} .
$$

Thus, equality (37) can be considered as the version, in the Fock-Bargmann space, of the Quantum Wick Theorem showed in [25] that works in the Fock space and with the related bosonic creation and annihilation operators of quantum field theory.

In the next, we provide a kind of quantum mean value formula for the time evolved $\widehat{G}(s):=U^{\dagger}(s) \widehat{G} U(s)$ where $U(s)=e^{-i \widehat{H} s}$ with $\widehat{H}$ as in (2) and $\widehat{G}=$ $\mathrm{Op}_{\mathrm{W}}(g)$ are Wick operators (see Sect. 3.1). This result will be applied within the proof of Theorem 1 for operators of type $\widehat{G}=\left(\hat{a}_{k}^{\dagger} \hat{a}_{k}+\frac{1}{N}\right)^{p}$ with creation and annihilation operators as in (3). This tool allows to avoid, in our setting and for our estimates, the well-known problem of Ehrenfest time, as well as to avoid the application of Grönwall Lemma (and thus exponential in time upper bounds) used in many papers on mean field estimates for NLS equations.

Proposition 2. Let $\widehat{G}=\mathrm{Op}_{\mathrm{W}}(g)$ be a Wick operator on $\mathcal{F}_{B}\left(\mathbb{C}^{L}\right)$ such that $g \in L^{1}\left(\mu_{N}\right)$. Let $\widehat{G}(s):=U^{\dagger}(s) \widehat{G} U(s)$ where $U(s)=e^{-i \widehat{H} s}$ with $\widehat{H}$ as in (2). Define $g(s, \bar{\omega}, \omega):=\left\langle\phi_{\omega}, \widehat{G}(s) \phi_{\omega}\right\rangle$. Then, $\forall s \geq 0$

$$
\int g(s, \bar{\omega}, \omega) d \mu_{N}(\bar{\omega}, \omega)=\int g(\bar{\omega}, \omega) d \mu_{N}(\bar{\omega}, \omega) .
$$

Proof. We apply Proposition 1

$$
\int g(\bar{\omega}, \omega) d \mu_{N}(\bar{\omega}, \omega)=\operatorname{Tr}\left(\frac{e^{-\lambda \widehat{N}}}{\gamma_{\lambda}} \mathrm{Op}_{\mathrm{W}}(g)\right), \quad \lambda>0
$$


and recall that the trace is invariant by unitary conjugations of operators, so that

$$
\operatorname{Tr}\left(\frac{e^{-\lambda \widehat{N}}}{\gamma_{\lambda}} \mathrm{Op}_{\mathrm{W}}(g)\right)=\operatorname{Tr}\left(U^{\dagger}(s) \frac{e^{-\lambda \widehat{N}}}{\gamma_{\lambda}} \mathrm{Op}_{\mathrm{W}}(g) U(s)\right) .
$$

Now we recall that $[\widehat{N}, \widehat{H}]=0$ and whence $\left[\widehat{N}, U^{\star}(s)\right]=0$, which gives

$$
\operatorname{Tr}\left(U^{\dagger}(s) \frac{e^{-\lambda \widehat{N}}}{\gamma_{\lambda}} \mathrm{Op}_{\mathrm{W}}(g) U(s)\right)=\operatorname{Tr}\left(\frac{e^{-\lambda \widehat{N}}}{\gamma_{\lambda}} U^{\dagger}(s) \mathrm{Op}_{\mathrm{W}}(g) U(s)\right)
$$

and applying again Proposition 1 for $\widehat{G}(s):=U^{\dagger}(s) \mathrm{Op}_{\mathrm{W}}(g) U(s)$ and, in view of Remark 2, we conclude

$$
\operatorname{Tr}\left(\frac{e^{-\lambda \widehat{N}}}{\gamma_{\lambda}} \widehat{G}(s)\right)=\int g(s, \bar{\omega}, \omega) d \mu_{N}(\bar{\omega}, \omega) .
$$

Remark 2. The Bose-Hubbard operator $\widehat{H}$ in (2) is self-adjoint on the Hilbert space $\mathcal{F}_{B}\left(\mathbb{C}^{L}\right)$ and thus, by the Stone Theorem, the $U(s):=e^{-i \widehat{H} s}$ is a one parameter group of unitary operators. Hence, $U(s)$ is bounded on $\mathcal{F}_{B}\left(\mathbb{C}^{L}\right)$ and this implies it is a Wick operator itself (see Sect. 3.1). It follows that $\widehat{G}(s):=U^{\dagger}(s) \mathrm{Op}_{\mathrm{W}}(g) U(s)$ equals a composition of Wick operators. Since the set of Wick operators is closed under composition, we deduce that $\widehat{G}(s)$ is still a Wick operator, and whence we denote its symbol by $g(s, \bar{\omega}, \omega)$.

Remark 3. The Proposition 2 works also with $g(s, a \bar{\omega}, a \omega)$ and $g(a \bar{\omega}, a \omega)$ for any fixed $a>0$. Indeed, for $\widetilde{N}:=N / a^{2}$ we have

$$
\begin{aligned}
\int g(s, a \bar{\omega}, a \omega) d \mu_{N}(\bar{\omega}, \omega) & =\int g(s, \bar{v}, v) d \mu_{\widetilde{N}}(\bar{v}, v)=\int g(\bar{v}, v) d \mu_{\widetilde{N}}(\bar{v}, v) \\
& =\int g(a \bar{\omega}, a \omega) d \mu_{N}(\bar{\omega}, \omega) .
\end{aligned}
$$

This observation will be useful in the application of this equality with $a=\sqrt{N}$.

We provide two technical lemma used in the next.

Lemma 1. Let $\mathcal{P}(\bar{\omega}, \omega)$ be as in (68), then for any $1 \leq p<\infty$ there exists a positive constant $C_{1, p}$ such that

$$
\left(\int \mathcal{P}(\bar{\omega}, \omega)^{4 p} d \mu_{N}\right)^{\frac{1}{4}} \leq C_{1, p}\left(\frac{L}{N}\right)^{p} .
$$

Moreover,

$$
\left(\int\left\langle\phi_{\sqrt{N} \omega},\left(\hat{a}_{k}^{\dagger}(0) \hat{a}_{k}(0)+\frac{1}{N}\right)^{2 p} \phi_{\sqrt{N} \omega}\right\rangle d \mu_{N}\right)^{\frac{1}{4}}=C_{2, p}\left(\frac{1}{\sqrt{N}}\right)^{p}
$$

for a positive constant $C_{2, p}$. 
Proof. . We first notice that for any fixed $\omega \in \mathbb{C}^{L}, \mathcal{P}(\bar{\omega}, \omega)$ is a sum of real nonnegative numbers

$$
\mathcal{P}(\bar{\omega}, \omega)=\sum_{j=1}^{L} f\left(\bar{\omega}_{j}, \omega_{j}\right)
$$

where $f\left(\bar{\omega}_{j}, \omega_{j}\right):=3 N\left|\omega_{j}\right|^{4}+4 \sqrt{N}\left|\omega_{j}\right|^{3}+\sqrt{2}\left|\omega_{j}\right|^{2}$, so by using Hölder inequality we get

$$
P(\bar{\omega}, \omega)^{4 p} \leq L^{4 p-1} \sum_{j} f\left(\bar{\omega}_{j}, \omega_{j}\right)^{4 p} .
$$

Since for any $v \in \mathbb{C}, f(\bar{v} / \sqrt{N}, v / \sqrt{N})=N^{-1} g(\bar{v}, v)$ for $g(\bar{v}, v)=3|v|^{4}+$ $4|v|^{3}+\sqrt{2}|v|^{2}$, integrating with respect to Gaussian measure and performing the change of variables $\omega_{j}^{\prime}=\sqrt{N} \omega_{j}$ we have

$$
\begin{aligned}
& \int_{\mathbb{C}^{L}} P(\bar{\omega}, \omega)^{4 p} d \mu_{N} \leq L^{4 p-1} \sum_{j} c_{N, L} \int_{\mathbb{C}^{L}} f\left(\bar{\omega}_{j}, \omega_{j}\right)^{4 p} e^{-N|\omega|^{2}} d \bar{\omega} \wedge d \omega \\
& =\frac{L^{4 p-1}}{N^{4 p}} \sum_{j} \frac{c_{N, L}}{N^{L}} \int_{\mathbb{C}^{L}}\left(3\left|\omega_{j}^{\prime}\right|^{4}+4\left|\omega_{j}^{\prime}\right|^{3}+\sqrt{2}\left|\omega_{j}^{\prime}\right|^{2}\right)^{4 p} e^{-\left|\omega^{\prime}\right|^{2}} d \bar{\omega}^{\prime} \wedge d \omega^{\prime} .
\end{aligned}
$$

For each $j=1, \ldots, L$, we factorize the integrals not containing $\omega_{j}$, so introducing the variable $v \in \mathbb{C}$ and its corresponding measure $d \bar{v} \wedge d v$ we have

$$
\begin{aligned}
& =\frac{L^{4 p-1}}{N^{4 p}} \sum_{j}\left(\int_{\mathbb{C}} e^{-|v|^{2}} d \bar{v} \wedge d v\right)^{L-1} \\
& \times\left(\int_{\mathbb{C}}\left(3|v|^{4}+4|v|^{3}+\sqrt{2}|v|^{2}\right)^{4 p} e^{-|v|^{2}} d \bar{v} \wedge d v\right) \\
& =\frac{L^{4 p}}{N^{4 p}} \int_{\mathbb{C}}\left(3|v|^{4}+4|v|^{3}+\sqrt{2}|v|^{2}\right)^{4 p} e^{-|v|^{2}} d \bar{v} \wedge d v
\end{aligned}
$$

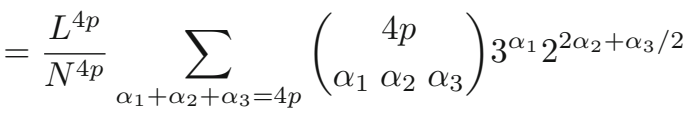

$$
\begin{aligned}
& \times \int_{\mathbb{C}}|v|^{4 \alpha_{1}+3 \alpha_{2}+2 \alpha_{3}} e^{-|v|^{2}} d \bar{v} \wedge d v \\
& =\frac{L^{4 p}}{N^{4 p}} \sum_{\alpha_{1}+\alpha_{2}+\alpha_{3}=4 p}\left(\begin{array}{c}
4 p \\
\alpha_{1} \alpha_{2} \alpha_{3}
\end{array}\right) 3^{\alpha_{1}} 2^{2 \alpha_{2}+\alpha_{3} / 2} \Gamma\left(2 \alpha_{1}+\frac{3}{2} \alpha_{2}+\alpha_{3}+1\right) \\
& =\frac{L^{4 p}}{N^{4 p}} \sum_{\alpha_{1}+\alpha_{2}+\alpha_{3}=4 p}\left(\begin{array}{c}
4 p \\
\alpha_{1} \alpha_{2} \alpha_{3}
\end{array}\right) 3^{\alpha_{1}} 2^{2 \alpha_{2}+\alpha_{3} / 2} \Gamma\left(2 \alpha_{1}+\frac{3}{2} \alpha_{2}+\alpha_{3}+1\right)
\end{aligned}
$$

where the Euler Gamma function has been introduced. Notice that in the first line we exploited the definition of $c_{N, L}=N^{L}$, while in the second line we used the fact that we have exactly $L$ equal integrals. Taking the fourth of root in 
the last expression, we get inequality (44) with

$$
C_{1, p}:=\left(\sum_{\alpha_{1}+\alpha_{2}+\alpha_{3}=4 p}\left(\begin{array}{c}
4 p \\
\alpha_{1} \alpha_{2} \alpha_{3}
\end{array}\right) 3^{\alpha_{1}} 2^{2 \alpha_{2}+\alpha_{3} / 2} \Gamma\left(2 \alpha_{1}+\frac{3}{2} \alpha_{2}+\alpha_{3}+1\right)\right)^{\frac{1}{4}} .
$$

To get (45), we need to compute the mean value $\left\langle\varphi_{\sqrt{N} \omega}, \hat{n}_{k}^{\alpha} \varphi_{\sqrt{N} \omega}\right\rangle=$ : $\left\langle\hat{n}_{k}^{\alpha}\right\rangle$ for any positive integer $\alpha$, where $\hat{n}_{k}=\hat{a}_{k}^{\dagger}(0) \hat{a}_{k}(0)$. We find that from the definition of Wick-* product there exists a recurrence relation between these quantities

$$
\left\langle\hat{n}_{k}^{\alpha}\right\rangle=\left(\left|\omega_{k}\right|^{2}+\frac{1}{N} \bar{\omega}_{k} \frac{\partial}{\partial \bar{\omega}_{k}}\right)\left\langle\hat{n}_{k}^{\alpha-1}\right\rangle=\left(\left|\omega_{k}\right|^{2}+\frac{1}{N} \bar{\omega}_{k} \frac{\partial}{\partial \bar{\omega}_{k}}\right)^{\alpha} \mathbf{1}
$$

where $\mathbf{1}$ is the constant function $\mathbf{1}(\bar{\omega}, \omega) \equiv 1$, so that in general

$$
\left\langle\hat{n}_{k}^{\alpha}\right\rangle=\sum_{\beta=1}^{\alpha} S(\alpha, \beta) \frac{\left|\omega_{k}\right|^{2 \beta}}{N^{\alpha-\beta}}
$$

where $S(\alpha, \beta)$ is the Stirling number of the second kind with integer parameters $\alpha$ and $\beta$ (see computations below). Since $\hat{n}_{k}$ and $N^{-1}$ commute as operators we can expand $\left(\hat{n}_{k}+N^{-1}\right)^{2 p}$ using the binomial theorem

$$
\begin{aligned}
\int_{\mathbb{C}^{L}}\left\langle\varphi_{\sqrt{N} \omega},\left(\hat{n}_{k}+\frac{1}{N}\right)^{2 p} \varphi_{\sqrt{N} \omega}\right\rangle d \mu_{N} \\
=\sum_{\alpha=1}^{2 p}\left(\begin{array}{c}
2 p \\
\alpha
\end{array}\right) N^{-2 p+\alpha} c_{N, L} \int_{\mathbb{C}^{L}}\left\langle\varphi_{\sqrt{N} \omega}, \hat{n}_{k}^{\alpha} \varphi_{\sqrt{N} \omega}\right\rangle e^{-N|\omega|^{2}} d \bar{\omega} \wedge d \omega \\
=\sum_{\alpha=1}^{2 p}\left(\begin{array}{c}
2 p \\
\alpha
\end{array}\right) \sum_{\beta=1}^{\alpha} S(\alpha, \beta) N^{-2 p+\beta} c_{N, L} \int_{\mathbb{C}^{L}}\left|\omega_{k}\right|^{2 \beta} e^{-N|\omega|^{2}} d \bar{\omega} \wedge d \omega \\
=N^{-2 p} \sum_{\alpha=1}^{2 p}\left(\begin{array}{c}
2 p \\
\alpha
\end{array}\right) \sum_{\beta=1}^{\alpha} S(\alpha, \beta) \beta ! .
\end{aligned}
$$

Taking again the fourth root, we get (45) with constant

$$
C_{2, p}:=\left(\sum_{\alpha=1}^{2 p}\left(\begin{array}{c}
2 p \\
\alpha
\end{array}\right) \sum_{\beta=1}^{\alpha} S(\alpha, \beta) \beta !\right)^{\frac{1}{4}}
$$

We now complete the proof of this lemma, showing that the coefficients of the polynomial in (52) are the Stirling number of the second kind (see [3], Par. 24.1.4 for their definition and properties). By the recurrence relation (51), we have 


$$
\begin{aligned}
\left\langle\hat{n}_{k}^{\alpha+1}\right\rangle & =\left(\left|\omega_{k}\right|^{2}+\frac{1}{N} \bar{\omega}_{k} \frac{\partial}{\partial \bar{\omega}_{k}}\right)\left\langle\hat{n}_{k}^{\alpha}\right\rangle \\
& =\left(\left|\omega_{k}\right|^{2}+\frac{1}{N} \bar{\omega}_{k} \frac{\partial}{\partial \bar{\omega}_{k}}\right) \sum_{\beta=1}^{\alpha} S(\alpha, \beta) \frac{\left|\omega_{k}\right|^{2 \beta}}{N^{\alpha-\beta}} \\
& =\sum_{\beta=1}^{\alpha} S(\alpha, \beta) \frac{\left|\omega_{k}\right|^{2 \beta+2}}{N^{\alpha-\beta}}+\sum_{\beta=1}^{\alpha} \beta S(\alpha, \beta) \frac{\left|\omega_{k}\right|^{2 \beta}}{N^{\alpha-\beta+1}} \\
& =\sum_{\beta=2}^{\alpha+1} S(\alpha, \beta-1) \frac{\left|\omega_{k}\right|^{2 \beta}}{N^{\alpha-\beta+1}}+\sum_{\beta=1}^{\alpha} \beta S(\alpha, \beta) \frac{\left|\omega_{k}\right|^{2 \beta}}{N^{\alpha-\beta+1}} \\
& =\sum_{\beta=1}^{\alpha+1}(S(\alpha, \beta-1)+\beta S(\alpha, \beta)) \frac{\left|\omega_{k}\right|^{2 \beta}}{N^{\alpha-\beta+1}},
\end{aligned}
$$

where we used the fact that $S(\alpha, \alpha)=S(\alpha, 1)=1$, as is easy verified using (51). Comparing the last expression with the general expansion of $\left\langle\hat{n}_{k}^{\alpha+1}\right\rangle$ as in (52) with exponent $\alpha+1$, we see that

$$
S(\alpha+1, \beta)=S(\alpha, \beta-1)+\beta S(\alpha, \beta)
$$

which is precisely the recurrence relation defining Stirling numbers.

Lemma 2. Let $\mathrm{Op}_{W}(g)$ be a Wick operator, $\rho(\bar{v}, v):=\left\langle\phi_{\sqrt{N} v}, \mathrm{Op}_{W}(g) \phi_{\sqrt{N} v}\right\rangle$. Then,

$$
\frac{\partial \rho}{\partial v_{j}}=\left\langle\left(\frac{\partial \phi_{\sqrt{N} v}^{\star}}{\partial v_{j}}\right)^{\star}, \mathrm{Op}_{W}(g) \phi_{\sqrt{N} v}\right\rangle+\left\langle\phi_{\sqrt{N} v}, \mathrm{Op}_{W}(g)\left(\frac{\partial \phi_{\sqrt{N} v}}{\partial v_{j}}\right)\right\rangle .
$$

Proof. We begin by

$$
\begin{aligned}
\frac{\partial \rho}{\partial v_{j}}= & \frac{\partial}{\partial v_{j}} \int \phi_{\sqrt{N} v}^{\star}(\bar{z}) \operatorname{Op}_{W}(g) \phi_{\sqrt{N} v}(\bar{z}) e^{-|z|^{2}} d z \wedge d \bar{z} \\
= & \int \frac{\partial}{\partial v_{j}} \phi_{\sqrt{N} v}^{\star}(\bar{z}) \cdot \mathrm{Op}_{W}(g) \phi_{\sqrt{N} v}(\bar{z}) e^{-|z|^{2}} d z \wedge d \bar{z} \\
& +\int \phi_{\sqrt{N} v}^{\star}(\bar{z}) \cdot \frac{\partial}{\partial v_{j}} \mathrm{Op}_{W}(g) \phi_{\sqrt{N} v}(\bar{z}) e^{-|z|^{2}} d z \wedge d \bar{z}
\end{aligned}
$$

In particular, the second term can be rewritten

$$
\begin{aligned}
& \int \phi_{\sqrt{N} v}^{\star}(\bar{z}) \cdot \frac{\partial}{\partial v_{j}} \mathrm{Op}_{W}(g) \phi_{\sqrt{N} v}(\bar{z}) e^{-|z|^{2}} d z \wedge d \bar{z} \\
& =\left.\left(\int \phi_{\sqrt{N} v}^{\star}(\bar{z}) \cdot \frac{\partial}{\partial w_{j}} \operatorname{Op}_{W}(g) \phi_{\sqrt{N} w}(\bar{z}) e^{-|z|^{2}} d z \wedge d \bar{z}\right)\right|_{w=v} \\
& =\left.\left(\frac{\partial}{\partial w_{j}} \int \phi_{\sqrt{N} v}^{\star}(\bar{z}) \cdot \mathrm{Op}_{W}(g) \phi_{\sqrt{N} w}(\bar{z}) e^{-|z|^{2}} d z \wedge d \bar{z}\right)\right|_{w=v} \\
& =\left.\left(\frac{\partial}{\partial w_{j}} \int\left(\mathrm{Op}_{W}(g)^{\dagger} \phi_{\sqrt{N} v}\right)^{\star}(\bar{z}) \cdot \phi_{\sqrt{N} w}(\bar{z}) e^{-|z|^{2}} d z \wedge d \bar{z}\right)\right|_{w=v} \\
& =\left.\left(\int\left(\operatorname{Op}_{W}(g)^{\dagger} \phi_{\sqrt{N} v}\right)^{\star}(\bar{z}) \cdot \frac{\partial}{\partial w_{j}} \phi_{\sqrt{N} w}(\bar{z}) e^{-|z|^{2}} d z \wedge d \bar{z}\right)\right|_{w=v}
\end{aligned}
$$




$$
=\int\left(\mathrm{Op}_{W}(g)^{\dagger} \phi_{\sqrt{N} v}\right)^{\star}(\bar{z}) \cdot \frac{\partial}{\partial v_{j}} \phi_{\sqrt{N} v}(\bar{z}) e^{-|z|^{2}} d z \wedge d \bar{z}
$$

and this last form equals $\left\langle\phi_{\sqrt{N} v}, \mathrm{Op}_{W}(g)\left(\frac{\partial \phi_{\sqrt{N} v}}{\partial v_{j}}\right)\right\rangle$.

In what follows, we get an estimate for $\left|\rho_{k}(t, \bar{\omega}, \omega)-u_{k}(t, \omega)\right|$ for any fixed $\omega \in \mathbb{C}^{L}$. This will be used, in the proof of Theorem 1 , to have the $L^{p}\left(\mu_{N}\right)$ estimate.

Proposition 3. Let $\Delta:=\left\{(\bar{\omega}, \omega) \mid \omega \in \mathbb{C}^{L}\right\} \subset \mathbb{C}^{2 L},\left(\bar{\Phi}_{t}, \Phi_{t}\right): \Delta \subset \mathbb{C}^{2 L} \rightarrow$ $\mathbb{C}^{2 L}$ the flow of $\dot{\gamma}=i\left(\partial_{\omega} \mathcal{H}(\gamma),-\partial_{\bar{\omega}} \mathcal{H}(\gamma)\right)$ with $\mathcal{H}$ as in $(6)$. Let $u(t, \omega):=$ $\left(u_{1}, \ldots u_{L}\right)(t, \omega)$ be the solution of $(1)$, and

$$
\begin{aligned}
\rho_{k}(t, \bar{\omega}, \omega) & :=\left\langle\phi_{\sqrt{N} \omega}, \hat{a}_{k}(t) \phi_{\sqrt{N} \omega}\right\rangle \\
n_{k}(t, \bar{\omega}, \omega) & :=\left\langle\phi_{\sqrt{N} \omega}, \hat{a}_{k}^{\dagger}(t) \hat{a}_{k}(t) \phi_{\sqrt{N} \omega}\right\rangle . \\
\mathcal{P}(\bar{v}, v) & :=\sum_{1 \leq j \leq L}\left[3 N\left|v_{j}\right|^{4}+4 \sqrt{N}\left|v_{j}\right|^{3}+\sqrt{2}\left|v_{j}\right|^{2}\right] .
\end{aligned}
$$

Then,

$$
\left|\rho_{k}(t, \bar{\omega}, \omega)-u_{k}(t, \omega)\right| \leq\left. U \int_{0}^{t} \mathcal{P}(\bar{v}, v)\left(n_{k}(s, \bar{v}, v)+\frac{1}{N}\right)^{\frac{1}{2}}\right|_{(\bar{v}, v)=\Phi_{t-s}(\bar{\omega}, \omega)} d s .
$$

Proof. The semigroup identity

$$
e^{-i N\left(\mathcal{L}_{1}+\mathcal{L}_{2}\right) t}=e^{-i N \mathcal{L}_{1} t}+\int_{0}^{t} e^{-i N \mathcal{L}_{1}(t-s)}(-i N) \mathcal{L}_{2} e^{-i N\left(\mathcal{L}_{1}+\mathcal{L}_{2}\right) s} d s
$$

applied to our case gives

$$
\rho_{k}(t, \bar{\omega}, \omega)-u_{k}(t, \omega)=\left.\int_{0}^{t}(-i N) \mathcal{L}_{2} \rho_{k}(s, \bar{v}, v)\right|_{(\bar{v}, v)=\Phi_{t-s}(\bar{\omega}, \omega)} d s,
$$

where the operator $\mathcal{L}_{2}$ reads

$$
\mathcal{L}_{2} \rho=\frac{1}{2} \frac{1}{N^{2}} \sum_{j=1}^{L}\left(\frac{\partial^{2} \rho}{\partial v_{j}^{2}} \frac{\partial^{2} \mathcal{H}}{\partial \bar{v}_{j}^{2}}-\frac{\partial \mathcal{H}}{\partial v_{j}^{2}} \frac{\partial^{2} \rho}{\partial \bar{v}_{j}^{2}}\right)
$$

and thus

$$
(-i N) \mathcal{L}_{2} \rho=(-i) \frac{U}{2 N} \sum_{j=1}^{L}\left(v_{j}^{2} \frac{\partial^{2} \rho}{\partial v_{j}^{2}}-\bar{v}_{j}^{2} \frac{\partial^{2} \rho}{\partial \bar{v}_{j}^{2}}\right) .
$$

We now recall the definition

$$
\rho_{k}(s, \bar{v}, v):=\left\langle\phi_{\sqrt{N} v}, \hat{a}_{k}(s) \phi_{\sqrt{N} v}\right\rangle
$$

where $\phi_{\sqrt{N} v}(\bar{z})=e^{\sqrt{N} v \bar{z}-\frac{1}{2} N|v|^{2}}$ and notice that

$$
\begin{aligned}
& \frac{\partial \phi_{\sqrt{N} v}}{\partial v_{j}}=\left(\sqrt{N} \bar{z}_{j}-\frac{N}{2} \bar{v}_{j}\right) \phi_{\sqrt{N} v}(\bar{z}), \\
& \frac{\partial \phi_{\sqrt{N} v}^{\star}}{\partial v_{j}}=-\frac{N}{2} \bar{v}_{j} \phi_{\sqrt{N} v}^{\star}(\bar{z}) .
\end{aligned}
$$


where $\phi_{\sqrt{N} v}^{\star}$ denotes the complex conjugated coherent state. Thanks to Lemma 2,

$$
\frac{\partial \rho_{k}}{\partial v_{j}}=\left\langle\left(\frac{\partial \phi_{\sqrt{N} v}^{\star}}{\partial v_{j}}\right)^{\star}, \hat{a}_{k}(s) \phi_{\sqrt{N} v}\right\rangle+\left\langle\phi_{\sqrt{N} v}, \hat{a}_{k}(s)\left(\frac{\partial \phi_{\sqrt{N} v}}{\partial v_{j}}\right)\right\rangle
$$

we have

$$
\begin{aligned}
\frac{\partial \rho_{k}}{\partial v_{j}} & =\left\langle-\frac{N}{2} v_{j} \phi_{\sqrt{N} v}, \hat{a}_{k}(s) \phi_{\sqrt{N} v}\right\rangle+\left\langle\phi_{\sqrt{N} v}, \hat{a}_{k}(s)\left(\sqrt{N} \bar{z}_{j}-\frac{N}{2} \bar{v}_{j}\right) \phi_{\sqrt{N} v}\right\rangle \\
& =-N \bar{v}_{j}\left\langle\phi_{\sqrt{N} v}, \hat{a}_{k}(s) \phi_{\sqrt{N} v}\right\rangle+\sqrt{N}\left\langle\phi_{\sqrt{N} v}, \hat{a}_{k}(s) \bar{z}_{j} \phi_{\sqrt{N} v}\right\rangle
\end{aligned}
$$

Notice that $\bar{z}_{j} \phi_{\sqrt{N} v}(\bar{z})=\sqrt{N} \hat{a}_{j}^{\dagger}(0) \phi_{\sqrt{N} v}(\bar{z})$ and thus

$$
\frac{\partial \rho_{k}}{\partial v_{j}}=-N \bar{v}_{j}\left\langle\phi_{\sqrt{N} v}, \hat{a}_{k}(s) \phi_{\sqrt{N} v}\right\rangle+N\left\langle\phi_{\sqrt{N} v}, \hat{a}_{k}(s) \hat{a}_{j}^{\dagger}(0) \phi_{\sqrt{N} v}\right\rangle .
$$

Applying twice this formula, we get

$$
\begin{aligned}
\frac{\partial^{2} \rho_{k}}{\partial v_{j}^{2}}= & N^{2} \bar{v}_{j}^{2}\left\langle\phi_{\sqrt{N} v}, \hat{a}_{k}(s) \phi_{\sqrt{N} v}\right\rangle-N^{2} \bar{v}_{j}\left\langle\phi_{\sqrt{N} v}, \hat{a}_{k}(s) \hat{a}_{j}^{\dagger}(0) \phi_{\sqrt{N} v}\right\rangle \\
& -N^{2} \bar{v}_{j}\left\langle\phi_{\sqrt{N} v}, \hat{a}_{k}(s) \hat{a}_{j}^{\dagger}(0) \phi_{\sqrt{N} v}\right\rangle+N^{2}\left\langle\phi_{\sqrt{N} v}, \hat{a}_{k}(s) \hat{a}_{j}^{\dagger}(0) \hat{a}_{j}^{\dagger}(0) \phi_{\sqrt{N} v}\right\rangle \\
= & N^{2} \bar{v}_{j}^{2}\left\langle\phi_{\sqrt{N} v}, \hat{a}_{k}(s) \phi_{\sqrt{N} v}\right\rangle-2 N^{2} \bar{v}_{j}\left\langle\phi_{\sqrt{N} v}, \hat{a}_{k}(s) \hat{a}_{j}^{\dagger}(0) \phi_{\sqrt{N} v}\right\rangle \\
& +N^{2}\left\langle\phi_{\sqrt{N} v}, \hat{a}_{k}(s) \hat{a}_{j}^{\dagger}(0) \hat{a}_{j}^{\dagger}(0) \phi_{\sqrt{N} v}\right\rangle .
\end{aligned}
$$

Applying the same computations for the derivatives on $\bar{v}_{j}$, we get

$$
\begin{aligned}
\frac{\partial^{2} \rho_{k}}{\partial \bar{v}_{j}^{2}}= & N^{2} v_{j}^{2}\left\langle\phi_{\sqrt{N} v}, \hat{a}_{k}(s) \phi_{\sqrt{N} v}\right\rangle-2 N^{2} v_{j}\left\langle\hat{a}_{j}^{\dagger}(0) \phi_{\sqrt{N} v}, \hat{a}_{k}(s) \phi_{\sqrt{N} v}\right\rangle \\
& +N^{2}\left\langle\hat{a}_{j}^{\dagger}(0) \hat{a}_{j}^{\dagger}(0) \phi_{\sqrt{N} v}, \hat{a}_{k}(s) \phi_{\sqrt{N} v}\right\rangle .
\end{aligned}
$$

The sum in (73) can now be rewritten as

$$
\begin{aligned}
\sum_{j=1}^{L} & \left(v_{j}^{2} \frac{\partial^{2} \rho_{k}}{\partial v_{j}^{2}}-\bar{v}_{j}^{2} \frac{\partial^{2} \rho_{k}}{\partial \bar{v}_{j}^{2}}\right) \\
= & \sum_{j=1}^{L} N^{2}\left|v_{j}\right|^{4}\left\langle\phi_{\sqrt{N} v}, \hat{a}_{k}(s) \phi_{\sqrt{N} v}\right\rangle-2 N^{2} v_{j}\left|v_{j}\right|^{2}\left\langle\phi_{\sqrt{N} v}, \hat{a}_{k}(s) \hat{a}_{j}^{\dagger}(0) \phi_{\sqrt{N} v}\right\rangle \\
& +N^{2} v_{j}^{2}\left\langle\phi_{\sqrt{N} v}, \hat{a}_{k}(s) \hat{a}_{j}^{\dagger}(0) \hat{a}_{j}^{\dagger}(0) \phi_{\sqrt{N} v}\right\rangle \\
& -\sum_{j=1}^{L} N^{2}\left|v_{j}\right|^{4}\left\langle\phi_{\sqrt{N} v}, \hat{a}_{k}(s) \phi_{\sqrt{N} v}\right\rangle-2 N^{2} \bar{v}_{j}\left|v_{j}\right|^{2}\left\langle\hat{a}_{j}^{\dagger}(0) \phi_{\sqrt{N} v}, \hat{a}_{k}(s) \phi_{\sqrt{N} v}\right\rangle \\
& +N^{2} \bar{v}_{j}^{2}\left\langle\hat{a}_{j}^{\dagger}(0) \hat{a}_{j}^{\dagger}(0) \phi_{\sqrt{N} v}, \hat{a}_{k}(s) \phi_{\sqrt{N} v}\right\rangle
\end{aligned}
$$


which simplifies to

$$
\begin{aligned}
\sum_{j=1}^{L} & \left(v_{j}^{2} \frac{\partial^{2} \rho_{k}}{\partial v_{j}^{2}}-\bar{v}_{j}^{2} \frac{\partial^{2} \rho_{k}}{\partial \bar{v}_{j}^{2}}\right) \\
= & \sum_{j=1}^{L}-2 N^{2} v_{j}\left|v_{j}\right|^{2}\left\langle\phi_{\sqrt{N} v}, \hat{a}_{k}(s) \hat{a}_{j}^{\dagger}(0) \phi_{\sqrt{N} v}\right\rangle \\
& +N^{2} v_{j}^{2}\left\langle\phi_{\sqrt{N} v}, \hat{a}_{k}(s) \hat{a}_{j}^{\dagger}(0) \hat{a}_{j}^{\dagger}(0) \phi_{\sqrt{N} v}\right\rangle \\
& +\sum_{j=1}^{L} 2 N^{2} \bar{v}_{j}\left|v_{j}\right|^{2}\left\langle\hat{a}_{j}^{\dagger}(0) \phi_{\sqrt{N} v}, \hat{a}_{k}(s) \phi_{\sqrt{N} v}\right\rangle \\
& -N^{2} \bar{v}_{j}^{2}\left\langle\hat{a}_{j}^{\dagger}(0) \hat{a}_{j}^{\dagger}(0) \phi_{\sqrt{N} v}, \hat{a}_{k}(s) \phi_{\sqrt{N} v}\right\rangle .
\end{aligned}
$$

The sum exhibits the following upper bound

$$
\begin{aligned}
& \left|\sum_{j=1}^{L}\left(v_{j}^{2} \frac{\partial^{2} \rho_{k}}{\partial v_{j}^{2}}-\bar{v}_{j}^{2} \frac{\partial^{2} \rho_{k}}{\partial \bar{v}_{j}^{2}}\right)\right| \\
& \leq \sum_{j=1}^{L} 2 N^{2}\left|v_{j}\right|^{3}\left\|\hat{a}_{k}^{\dagger}(s) \phi_{\sqrt{N} v}\right\|\left\|\hat{a}_{j}^{\dagger}(0) \phi_{\sqrt{N} v}\right\| \\
& +N^{2}\left|v_{j}\right|^{2}\left\|\hat{a}_{k}^{\dagger}(s) \phi_{\sqrt{N} v}\right\|\left\|\hat{a}_{j}^{\dagger}(0) \hat{a}_{j}^{\dagger}(0) \phi_{\sqrt{N} v}\right\| \\
& \quad+\sum_{j=1}^{L} 2 N^{2}\left|v_{j}\right|^{3}\left\|\hat{a}_{j}^{\dagger}(0) \phi_{\sqrt{N} v}\right\|\left\|\hat{a}_{k}(s) \phi_{\sqrt{N} v}\right\| \\
& \quad+N^{2}\left|v_{j}\right|^{2}\left\|\hat{a}_{j}^{\dagger}(0) \hat{a}_{j}^{\dagger}(0) \phi_{\sqrt{N} v}\right\|\left\|\hat{a}_{k}(s) \phi_{\sqrt{N} v}\right\|,
\end{aligned}
$$

namely

$$
\begin{aligned}
\leq & \sum_{j=1}^{L}\left(2 N^{2}\left|v_{j}\right|^{3}\left\|\hat{a}_{j}^{\dagger}(0) \phi_{\sqrt{N} v}\right\|+N^{2}\left|v_{j}\right|^{2}\left\|\hat{a}_{j}^{\dagger}(0) \hat{a}_{j}^{\dagger}(0) \phi_{\sqrt{N} v}\right\|\right)\left\|\hat{a}_{k}^{\dagger}(s) \phi_{\sqrt{N} v}\right\| \\
& +\sum_{j=1}^{L}\left(2 N^{2}\left|v_{j}\right|^{3}\left\|\hat{a}_{j}^{\dagger}(0) \phi_{\sqrt{N} v}\right\|+N^{2}\left|v_{j}\right|^{2}\left\|\hat{a}_{j}^{\dagger}(0) \hat{a}_{j}^{\dagger}(0) \phi_{\sqrt{N} v}\right\|\right)\left\|\hat{a}_{k}(s) \phi_{\sqrt{N} v}\right\| .
\end{aligned}
$$

We need to get an estimate for $\left\|\hat{a}_{j}^{\dagger}(0) \phi_{\sqrt{N} v}\right\|$ and $\left\|\hat{a}_{j}^{\dagger}(0) \hat{a}_{j}^{\dagger}(0) \phi_{\sqrt{N} v}\right\|$.

$$
\begin{aligned}
\left\|\hat{a}_{j}^{\dagger}(0) \phi_{\sqrt{N} v}\right\|^{2} & =\left\langle\phi_{\sqrt{N} v}, \hat{a}_{k}(0) \hat{a}_{j}^{\dagger}(0) \phi_{\sqrt{N} v}\right\rangle=\left\langle\phi_{\sqrt{N} v},\left(\hat{a}_{j}^{\dagger}(0) \hat{a}_{j}(0)+\frac{1}{N}\right) \phi_{\sqrt{N} v}\right\rangle \\
& =\left\langle\phi_{\sqrt{N} v}, \hat{a}_{j}^{\dagger}(0) \hat{a}_{j}(0) \phi_{\sqrt{N} v}\right\rangle+\frac{1}{N} .
\end{aligned}
$$

Since $\hat{a}_{j}(0) \phi_{\sqrt{N} v}=v_{j} \phi_{\sqrt{N} v}$ and recalling that $\phi_{\sqrt{N} v}$ are normalized, it follows

$$
\begin{aligned}
\left\|\hat{a}_{j}^{\dagger}(0) \phi_{\sqrt{N} v}\right\|^{2} & =\left\langle\phi_{\sqrt{N} v}, \hat{a}_{j}(0) \hat{a}_{j}^{\dagger}(0) \phi_{\sqrt{N} v}\right\rangle=\left\langle\phi_{\sqrt{N} v},\left(\hat{a}_{j}^{\dagger}(0) \hat{a}_{j}(0)+\frac{1}{N}\right) \phi_{\sqrt{N} v}\right\rangle \\
& =\left\langle\phi_{\sqrt{N} v}, \hat{a}_{j}^{\dagger}(0) \hat{a}_{j}(0) \phi_{\sqrt{N} v}\right\rangle+\frac{1}{N}=\left|v_{j}\right|^{2}+\frac{1}{N},
\end{aligned}
$$


and thus

$$
\left\|\hat{a}_{j}^{\dagger}(0) \phi_{\sqrt{N} v}\right\|=\left(\left|v_{j}\right|^{2}+\frac{1}{N}\right)^{\frac{1}{2}} \leq\left|v_{j}\right|+\frac{1}{\sqrt{N}} .
$$

We now look at

$$
\begin{aligned}
\left\|\hat{a}_{j}^{\dagger}(0) \hat{a}_{j}^{\dagger}(0) \phi_{\sqrt{N} v}\right\|^{2}=\left\langle\phi_{\sqrt{N} v}, \hat{a}_{j}(0) \hat{a}_{j}(0) \hat{a}_{j}^{\dagger}(0) \hat{a}_{j}^{\dagger}(0) \phi_{\sqrt{N} v}\right\rangle \\
=\left\langle\phi_{\sqrt{N} v}, \hat{a}_{j}(0)\left(\hat{a}_{j}^{\dagger}(0) \hat{a}_{j}(0)+\frac{1}{N}\right) \hat{a}_{j}^{\dagger}(0) \phi_{\sqrt{N} v}\right\rangle \\
=\left\langle\phi_{\sqrt{N} v}, \hat{a}_{j}(0) \hat{a}_{j}^{\dagger}(0) \hat{a}_{j}(0) \hat{a}_{j}^{\dagger}(0) \phi_{\sqrt{N} v}\right\rangle+\frac{1}{N}\left\|\hat{a}_{j}^{\dagger}(0) \phi_{\sqrt{N} v}\right\|^{2} \\
=\left\langle\phi_{\sqrt{N} v},\left(\hat{a}_{j}^{\dagger}(0) \hat{a}_{j}(0)+\frac{1}{N}\right)\left(\hat{a}_{j}^{\dagger}(0) \hat{a}_{j}(0)+\frac{1}{N}\right) \phi_{\sqrt{N} v}\right\rangle+\frac{1}{N}\left\|\hat{a}_{j}^{\dagger}(0) \phi_{\sqrt{N} v}\right\|^{2} \\
=\left\|\hat{a}_{j}^{\dagger}(0) \hat{a}_{j}(0) \phi_{\sqrt{N} v}\right\|^{2}+\frac{2}{N}\left\langle\phi_{\sqrt{N} v}, \hat{a}_{j}^{\dagger}(0) \hat{a}_{j}(0) \phi_{\sqrt{N} v}\right\rangle \\
\quad+\frac{1}{N^{2}}+\frac{1}{N}\left\|\hat{a}_{j}^{\dagger}(0) \phi_{\sqrt{N} v}\right\|^{2} .
\end{aligned}
$$

By using again $\hat{a}_{j}(0) \phi_{\sqrt{N} v}=v_{j} \phi_{\sqrt{N} v}$ and (89), we have

$$
\begin{aligned}
& \left\|\hat{a}_{j}^{\dagger}(0) \hat{a}_{j}^{\dagger}(0) \phi \sqrt{N} v\right\|^{2} \\
& \quad=\left|v_{j}\right|^{2}\left(\left|v_{j}\right|^{2}+\frac{1}{N}\right)+\frac{2}{N}\left|v_{j}\right|^{2}+\frac{1}{N^{2}}+\frac{1}{N}\left(\left|v_{j}\right|^{2}+\frac{1}{N}\right) \\
& \quad=\left|v_{j}\right|^{4}+\frac{4}{N}\left|v_{j}\right|^{2}+\frac{2}{N^{2}},
\end{aligned}
$$

and hence

$$
\left\|\hat{a}_{j}^{\dagger}(0) \hat{a}_{j}^{\dagger}(0) \phi_{\sqrt{N} v}\right\| \leq\left|v_{j}\right|^{2}+\frac{2}{\sqrt{N}}\left|v_{j}\right|+\frac{\sqrt{2}}{N} .
$$

Inserting (90)-(93) into (88), we get

$$
\begin{aligned}
& \left|\sum_{j=1}^{L}\left(v_{j}^{2} \frac{\partial^{2} \rho_{k}}{\partial v_{j}^{2}}-\bar{v}_{j}^{2} \frac{\partial^{2} \rho_{k}}{\partial \bar{v}_{j}^{2}}\right)\right| \leq \sum_{j=1}^{L}\left(2 N^{2}\left|v_{j}\right|^{3}\left(\left|v_{j}\right|+\frac{1}{\sqrt{N}}\right)\right. \\
& \left.+N^{2}\left|v_{j}\right|^{2}\left(\left|v_{j}\right|^{2}+\frac{2}{\sqrt{N}}\left|v_{j}\right|+\frac{\sqrt{2}}{N}\right)\right)\left\|\hat{a}_{k}^{\dagger}(s) \phi_{\sqrt{N} v}\right\| \\
& \quad+\sum_{j=1}^{L}\left(2 N^{2}\left|v_{j}\right|^{3}\left(\left|v_{j}\right|+\frac{1}{\sqrt{N}}\right)\right. \\
& \left.+N^{2}\left|v_{j}\right|^{2}\left(\left|v_{j}\right|^{2}+\frac{2}{\sqrt{N}}\left|v_{j}\right|+\frac{\sqrt{2}}{N}\right)\right)\left\|\hat{a}_{k}(s) \phi_{\sqrt{N} v}\right\| .
\end{aligned}
$$

Thus, 


$$
\begin{aligned}
& \left|\sum_{j=1}^{L}\left(v_{j}^{2} \frac{\partial^{2} \rho_{k}}{\partial v_{j}^{2}}-\bar{v}_{j}^{2} \frac{\partial^{2} \rho_{k}}{\partial \bar{v}_{j}^{2}}\right)\right| \\
& \leq N^{2} \sum_{j=1}^{L}\left(3\left|v_{j}\right|^{4}+\frac{4}{\sqrt{N}}\left|v_{j}\right|^{3}+\frac{\sqrt{2}}{N}\left|v_{j}\right|^{2}\right)\left(\left\|\hat{a}_{k}^{\dagger}(s) \phi_{\sqrt{N} v}\right\|+\left\|\hat{a}_{k}(s) \phi_{\sqrt{N} v}\right\|\right) .
\end{aligned}
$$

We observe that

$$
\begin{aligned}
\left\|\hat{a}_{k}(s) \phi_{\sqrt{N} v}\right\| & =\left(\left\langle\phi_{\sqrt{N} v}, \hat{a}_{k}^{\dagger}(s) \hat{a}_{k}(s) \phi_{\sqrt{N} v}\right\rangle\right)^{\frac{1}{2}} \\
& \leq\left(\left\langle\phi_{\sqrt{N} v}, \hat{a}_{k}^{\dagger}(s) \hat{a}_{k}(s) \phi_{\sqrt{N} v}\right\rangle+\frac{1}{N}\right)^{\frac{1}{2}}
\end{aligned}
$$

and

$$
\begin{aligned}
\left\|\hat{a}_{k}^{\dagger}(s) \phi_{\sqrt{N} v}\right\| & =\left(\left\langle\phi_{\sqrt{N} v}, \hat{a}_{k}(s) \hat{a}_{k}^{\dagger}(s) \phi_{\sqrt{N} v}\right\rangle\right)^{\frac{1}{2}} \\
& =\left(\left\langle\phi_{\sqrt{N} v}, \hat{a}_{k}^{\dagger}(s) \hat{a}_{k}(s) \phi_{\sqrt{N} v}\right\rangle+\frac{1}{N}\right)^{\frac{1}{2}} .
\end{aligned}
$$

As a consequence,

$$
\begin{aligned}
& \left|\sum_{j=1}^{L}\left(v_{j}^{2} \frac{\partial^{2} \rho_{k}}{\partial v_{j}^{2}}-\bar{v}_{j}^{2} \frac{\partial^{2} \rho_{k}}{\partial \bar{v}_{j}^{2}}\right)\right| \\
& \quad \leq 2 N^{2} \sum_{j=1}^{L}\left(3\left|v_{j}\right|^{4}+\frac{4}{\sqrt{N}}\left|v_{j}\right|^{3}+\frac{\sqrt{2}}{N}\left|v_{j}\right|^{2}\right)\left(n_{k}(s, \bar{v}, v)+\frac{1}{N}\right)^{\frac{1}{2}} .
\end{aligned}
$$

Now define $\mathcal{P}(\bar{v}, v):=\sum_{1 \leq j \leq L}\left(3 N\left|v_{j}\right|^{4}+4 \sqrt{N}\left|v_{j}\right|^{3}+\sqrt{2}\left|v_{j}\right|^{2}\right)$ and recall equalities (71)-(73) which imply the statement (69).

In view of previous propositions, we can now provide the proof of the main result of the paper.

Proof of Theorem 1. Recalling (69), we define the positive function

$$
\psi(s):=\left.U \mathcal{P}(\bar{v}, v)\left(n_{k}(s, \bar{v}, v)+\frac{1}{N}\right)^{\frac{1}{2}}\right|_{(\bar{v}, v)=\Phi_{t-s}(\bar{\omega}, \omega)},
$$

and for the sake of simplicity we avoid to write the dependence on $(\bar{\omega}, \omega)$. Thus, $\left|\rho_{k}-u_{k}\right|(t) \leq \int_{0}^{t} \psi(s) d s$ and

$$
\left\|\rho_{k}(t)-u_{k}(t)\right\|_{L^{p}\left(\mu_{N}\right)} \leq\left\|\int_{0}^{t} \psi(s) d s\right\|_{L^{p}\left(\mu_{N}\right)} .
$$

More in details,

$$
\left\|\int_{0}^{t} \psi(s) d s\right\|_{L^{p}\left(\mu_{N}\right)}^{p}=\int\left(\int_{0}^{t} \psi(s) d s\right)^{p} d \mu_{N} .
$$

The Hölder inequality $\|f g\|_{L^{1}} \leq\|f\|_{L^{p}}\|g\|_{L^{q}}$ with $1 / q+1 / p=1$, allows

$$
\int_{0}^{t} \psi(s) d s \leq\left(\int_{0}^{t} \psi^{p}(s) d s\right)^{\frac{1}{p}} t^{1-\frac{1}{p}}
$$


and hence

$$
\left(\int_{0}^{t} \psi(s) d s\right)^{p} \leq \int_{0}^{t} \psi^{p}(s) d s t^{p-1}
$$

This gives

$$
\int\left(\int_{0}^{t} \psi(s) d s\right)^{p} d \mu_{N} \leq t^{p-1} \int_{0}^{t}\left(\int \psi^{p}(s) d \mu_{N}\right) d s
$$

We now focus our attention to

$$
\int \psi^{p}(s) d \mu_{N}=\int\left(\left.U \mathcal{P}(\bar{v}, v)\left(n_{k}(s, \bar{v}, v)+\frac{1}{N}\right)^{\frac{1}{2}}\right|_{(\bar{v}, v)=\Phi_{t-s}(\bar{\omega}, \omega)}\right)^{p} d \mu_{N} .
$$

The invariance of $\mu_{N}$ under the flow $\Phi_{t-s}$ implies

$$
\begin{aligned}
\int \psi^{p}(s) d \mu_{N} & =\int\left(U \mathcal{P}(\bar{\omega}, \omega)\left(n_{k}(s, \bar{\omega}, \omega)+\frac{1}{N}\right)^{\frac{1}{2}}\right)^{p} d \mu_{N} \\
& =\int\left(U^{2} \mathcal{P}(\bar{\omega}, \omega)^{2}\left(n_{k}(s, \bar{\omega}, \omega)+\frac{1}{N}\right)\right)^{\frac{p}{2}} d \mu_{N} \\
& =\int\left(\left\langle\phi_{\sqrt{N} \omega}, B_{k}(s) \phi_{\sqrt{N} \omega}\right\rangle\right)^{\frac{p}{2}} d \mu_{N}
\end{aligned}
$$

where we have just defined the positive definite operator

$$
B_{k}(s):=U^{2} \mathcal{P}(\bar{\omega}, \omega)^{2}\left(\hat{a}_{k}^{\dagger}(s) \hat{a}_{k}(s)+\frac{1}{N}\right) .
$$

Now assume that $p=2^{m}$ with $m \in \mathbb{N}$ so that

$$
\left(\left\langle\phi_{\sqrt{N} \omega}, B_{k}(s) \phi_{\sqrt{N} \omega}\right\rangle\right)^{\frac{p}{2}} \leq\left\langle\phi_{\sqrt{N} \omega}, B_{k}^{p}(s) \phi_{\sqrt{N} \omega}\right\rangle^{\frac{1}{2}} .
$$

We get, thanks to the normalization of $\mu_{N}$,

$$
\begin{aligned}
\int \psi^{p}(s) d \mu_{N} & \leq \int\left\langle\phi_{\sqrt{N} \omega}, B_{k}^{p}(s) \phi_{\sqrt{N} \omega}\right\rangle^{\frac{1}{2}} d \mu_{N} \\
& \leq\left(\int\left\langle\phi_{\sqrt{N} \omega}, B_{k}^{p}(s) \phi_{\sqrt{N} \omega}\right\rangle d \mu_{N}\right)^{\frac{1}{2}}
\end{aligned}
$$

and recalling (109),

$$
\int \psi^{p}(s) d \mu_{N} \leq\left(\int U^{2 p} \mathcal{P}(\bar{\omega}, \omega)^{2 p}\left\langle\phi_{\sqrt{N} \omega},\left(\hat{a}_{k}^{\dagger}(s) \hat{a}_{k}(s)+\frac{1}{N}\right)^{p} \phi_{\sqrt{N} \omega}\right\rangle d \mu_{N}\right)^{\frac{1}{2}} .
$$

The Cauchy-Schwarz inequality gives

$$
\leq\left(\int U^{4 p} \mathcal{P}(\bar{\omega}, \omega)^{4 p} d \mu_{N}\right)^{\frac{1}{4}}\left(\int\left\langle\phi_{\sqrt{N} \omega},\left(\hat{a}_{k}^{\dagger}(s) \hat{a}_{k}(s)+\frac{1}{N}\right)^{p} \phi_{\sqrt{N} \omega}\right\rangle^{2} d \mu_{N}\right)^{\frac{1}{4}} .
$$

Since $\hat{a}_{k}^{\dagger}(s) \hat{a}_{k}(s)+\frac{1}{N}$ is positive definite, we have the upper bound 


$$
\leq\left(\int U^{4 p} \mathcal{P}(\bar{\omega}, \omega)^{4 p} d \mu_{N}\right)^{\frac{1}{4}}\left(\int\left\langle\phi_{\sqrt{N} \omega},\left(\hat{a}_{k}^{\dagger}(s) \hat{a}_{k}(s)+\frac{1}{N}\right)^{2 p} \phi_{\sqrt{N} \omega}\right\rangle d \mu_{N}\right)^{\frac{1}{4}}
$$

Observe that, since $U^{\star}(s) U(s)=\mathrm{Id}$,

$$
\left(\hat{a}_{k}^{\dagger}(s) \hat{a}_{k}(s)+\frac{1}{N}\right)^{2 p}=U^{\star}(s)\left(\hat{a}_{k}^{\dagger} \hat{a}_{k}+\frac{1}{N}\right)^{2 p} U(s) .
$$

Now apply Proposition 2 and Remark 3 in order to rewrite (114) as

$$
=\left(\int U^{4 p} \mathcal{P}(\bar{\omega}, \omega)^{4 p} d \mu_{N}\right)^{\frac{1}{4}}\left(\int\left\langle\phi_{\sqrt{N} \omega},\left(\hat{a}_{k}^{\dagger}(0) \hat{a}_{k}(0)+\frac{1}{N}\right)^{2 p} \phi_{\sqrt{N} \omega}\right\rangle d \mu_{N}\right)^{\frac{1}{4}} .
$$

Integrating these terms, see Lemma 1 , we have two constants $C_{1, p}$ and $C_{2, p}>0$ such that

$$
\left(\int \mathcal{P}(\bar{\omega}, \omega)^{4 p} d \mu_{N}\right)^{\frac{1}{4}} \leq C_{1, p}\left(\frac{L}{N}\right)^{p}
$$

and

$$
\left(\int\left\langle\phi_{\sqrt{N} \omega},\left(\hat{a}_{k}^{\dagger}(0) \hat{a}_{k}(0)+\frac{1}{N}\right)^{2 p} \phi_{\sqrt{N} \omega}\right\rangle d \mu_{N}\right)^{\frac{1}{4}}=C_{2, p}\left(\frac{1}{\sqrt{N}}\right)^{p} .
$$

Thus,

$$
\int \psi^{p}(s) d \mu_{N} \leq U^{p} C_{1, p}\left(\frac{L}{N}\right)^{p} C_{2, p}\left(\frac{1}{\sqrt{N}}\right)^{p} .
$$

We are now in the position to conclude

$$
\begin{aligned}
\left\|\rho_{k}(t)-u_{k}(t)\right\|_{L^{p}\left(\mu_{N}\right)}^{p} & \leq t^{p-1} \int_{0}^{t} U^{p} C_{1, p} C_{2, p}\left(\frac{1}{\sqrt{N}}\right)^{p} d s \\
& =t^{p} U^{p} C_{1, p}\left(\frac{L}{N}\right)^{p} C_{2, p}\left(\frac{1}{\sqrt{N}}\right)^{p},
\end{aligned}
$$

so that by defining

$$
B_{p}:=\left(C_{1, p} C_{2, p}\right)^{\frac{1}{p}},
$$

we have, in the case $p=2^{m}$ with $m \in \mathbb{N}$,

$$
\left\|\rho_{k}(t)-u_{k}(t)\right\|_{L^{p}\left(\mu_{N}\right)} \leq t U B_{p} \frac{L}{N} \frac{1}{\sqrt{N}} .
$$

Now observe that, thanks to normalization of $\mu_{N}$ and a simple application of Hölder inequality, we have $\left\|\rho_{k}(t)-u_{k}(t)\right\|_{L^{p}\left(\mu_{N}\right)} \leq\left\|\rho_{k}(t)-u_{k}(t)\right\|_{L^{\alpha}\left(\mu_{N}\right)}$ for any $\alpha \geq p$. Thus, fix $\alpha:=2^{p}$ so that

$$
A_{p}:=B_{2^{p}},
$$

ensures now for all $p \geq 1$ the inequality

$$
\left\|\rho_{k}(t)-u_{k}(t)\right\|_{L^{p}\left(\mu_{N}\right)} \leq t U A_{p} \frac{L}{N} \frac{1}{\sqrt{N}} .
$$


It remains to prove that $\rho_{k}, u_{k} \in L^{p}\left(\mu_{N}\right)$. Recall that $u_{k}(t, \omega)=\Phi_{t}^{(k)}(\bar{\omega}, \omega)$ and that $\mu_{N}$ is invariant under $\Phi_{t}$. Hence,

$$
\begin{aligned}
& \int\left|u_{k}(t, \omega)\right|^{p} d \mu_{N}(\bar{\omega}, \omega)=\int\left|\Phi_{t}^{(k)}(\bar{\omega}, \omega)\right|^{p} d \mu_{N}(\bar{\omega}, \omega) \\
= & \int\left|\omega^{k}\right|^{p}\left(\Phi_{t}\right)_{\star} d \mu_{N}(\bar{\omega}, \omega)=\int\left|\omega^{k}\right|^{p} d \mu_{N}(\bar{\omega}, \omega)<+\infty .
\end{aligned}
$$

where the last inequality is guaranteed since $\mu_{N}$ is a Gaussian type measure and $\left|\omega^{k}\right|^{p}$ is a polynomial term. Inequality (125) gives $\left\|\rho_{k}(t)-u_{k}(t)\right\|_{L^{p}\left(\mu_{N}\right)}<$ $+\infty$ and thus $\left\|\rho_{k}\right\|_{L^{p}\left(\mu_{N}\right)}<+\infty$.

An immediate consequence of Theorem 1 is the next corollary.

Proof of Corollary 1. Let $0<\epsilon<\frac{1}{2}$ and define the set

$$
\Omega_{k}:=\left\{(\omega, \bar{\omega})|\quad| \rho_{k}-u_{k} \mid(t, \omega, \bar{\omega})>A_{p} \frac{L}{N} \frac{U t}{N^{\epsilon}}, \quad \forall t \geq 0\right\} .
$$

Then,

$$
\begin{aligned}
\mu_{N}\left(\Omega_{k}\right)\left(A_{p} \frac{L}{N} \frac{U t}{N^{\epsilon}}\right)^{p} & <\int_{\Omega_{k}}\left|\rho_{k}-u_{k}\right|^{p}(t, \omega, \bar{\omega}) d \mu_{N}(\bar{\omega}, \omega) \\
& \leq \int\left|\rho_{k}-u_{k}\right|^{p}(t, \omega, \bar{\omega}) d \mu_{N}(\bar{\omega}, \omega) .
\end{aligned}
$$

Recalling inequality (125), we get

$$
\mu_{N}\left(\Omega_{k}\right)\left(A_{p} \frac{L}{N} \frac{U t}{N^{\epsilon}}\right)^{p} \leq\left(A_{p} \frac{L}{N} \frac{U t}{\sqrt{N}}\right)^{p}
$$

hence

$$
\mu_{N}\left(\Omega_{k}\right) \leq N^{-p \cdot\left(\frac{1}{2}-\epsilon\right)}, \quad \forall p \geq 1 \quad \forall N \geq 1
$$

\section{Acknowledgements}

We thank Alberto Maiocchi (University of Padova), with whom we are currently writing another paper on the subject, for important suggestions on the use of the measure. We are also very much grateful to Riccardo Adami, Dario Bambusi and Andrea Sacchetti for useful comments on the results.

Funding Open access funding provided by Universitá degli Studi di Padova within the CRUI-CARE Agreement.

\section{Declarations}

Conflict of interest The authors declare that they have no conflict of interest. 
Open Access. This article is licensed under a Creative Commons Attribution 4.0 International License, which permits use, sharing, adaptation, distribution and reproduction in any medium or format, as long as you give appropriate credit to the original author(s) and the source, provide a link to the Creative Commons licence, and indicate if changes were made. The images or other third party material in this article are included in the article's Creative Commons licence, unless indicated otherwise in a credit line to the material. If material is not included in the article's Creative Commons licence and your intended use is not permitted by statutory regulation or exceeds the permitted use, you will need to obtain permission directly from the copyright holder. To view a copy of this licence, visit http://creativecommons. org/licenses/by/4.0/.

Publisher's Note Springer Nature remains neutral with regard to jurisdictional claims in published maps and institutional affiliations.

\section{Appendix}

\subsection{Remarks on Scaling, Model and Relevant Quantities}

Here, we provide some remarks on the $N$-scaling in the many-body operator (2) and its modifications. One can also define, in place of (2),

$$
\widehat{H}:=\sum_{1 \leq j \leq L}\left[E \hat{b}_{j}^{\dagger} \hat{b}_{j}+J\left(\hat{b}_{j+1}^{\dagger} \hat{b}_{j}+\hat{b}_{j}^{\dagger} \hat{b}_{j+1}\right)+\frac{U}{2 N^{\alpha}} \hat{b}_{j}^{\dagger} \hat{b}_{j}^{\dagger} \hat{b}_{j} \hat{b}_{j}\right]
$$

where the coefficient $1 / N^{\alpha}$ with $0<\alpha \leq 1$ (in place of $1 / N$ ) multiplies the quartic term of the operator. Notice that the parameter $\alpha>0$ can be arbitrarily small, and thus the operator of the quartic part has a "weight" which is close to have the same order of the quadratic part. In this setting, we can choose the rescaling of the bosonic operators $\hat{a}_{k}:=\hat{b}_{k} / \sqrt{N^{\alpha}}$ and commutation rules becomes $\left[\hat{a}_{k}, \hat{a}_{\mu}^{\dagger}\right]=\delta_{k \mu} \mathrm{Id} / N^{\alpha}$. The related Heisenberg Eq. (4) for $\hat{a}_{k}$ is not modified, and thus the DNLS Eq. (1) is not changed and reads

$$
i \frac{d}{d t} u_{k}(t)=E u_{k}(t)+J\left(u_{k+1}(t)+u_{k-1}(t)\right)+U\left|u_{k}(t)\right|^{2} u_{k}(t)
$$

where we underline that here the cubic part has the same order of the linear part and does not depend on $N$ or the exponent $\alpha$. The $L^{p}$-estimate in Theorem 1 thus becomes

$$
\left\|\rho_{\alpha, k}(t)-u_{k}(t)\right\|_{L^{p}\left(\mu_{\alpha, N}\right)} \leq A_{p} \frac{L}{N^{\alpha}} \frac{U t}{N^{\alpha / 2}}
$$

for $\rho_{\alpha, k}(t, \bar{\omega}, \omega):=\left\langle\phi_{\sqrt{N^{\alpha}} \omega}, \hat{a}_{k}(t) \phi_{\sqrt{N^{\alpha}} \omega}\right\rangle$ and $\mu_{\alpha, N}:=\mu_{N^{\alpha}}$. When $\alpha=1$ this estimate recovers the one of Theorem 1 within the original setting of the many-body operator (2).

Concerning possible extensions of the model, we can also consider the more general operator

$$
\widehat{H}:=\sum_{1 \leq k, j \leq L}\left[\tau_{k j} \hat{b}_{k}^{\dagger} \hat{b}_{j}+\frac{1}{2 N} \sum_{1 \leq r, s \leq L} U_{k j}^{r s} \hat{b}_{k}^{\dagger} \hat{b}_{r}^{\dagger} \hat{b}_{j} \hat{b}_{s}\right]
$$


under for example the assumption that $\left|U_{k j}^{r s}\right| \leq \bar{U}$. Indeed, in this more general case the dependence from the parameter $L$ into the $L^{p}$-estimate of Theorem 1 will be $L^{4} / N$ in place of $L / N$, namely

$$
\left\|\rho_{k}(t)-u_{k}(t)\right\|_{L^{p}\left(\mu_{N}\right)} \leq A_{p} \frac{L^{4}}{N} \frac{\bar{U} t}{\sqrt{N}},
$$

where the constant $A_{p}$ will be changed by a more general formula.

In the current paper, we have considered the most simple setting involving the quantum dynamics driven by $\widehat{H}$ as in (2) in order to make more clear and direct the exposition of our approach of mean field estimates for Wick operators on Bargmann space.

Moreover, concerning our focussing on the dynamics of $\left\langle a_{k}\right\rangle$ as the main physical quantity, we stress that along the same lines, we can treat the dynamics of polynomials in $a_{k}$ and $a_{k}^{\dagger}$. For example, the quantity

$$
\widetilde{\rho}_{j k}(t, \bar{\omega}, \omega):=\left\langle\phi_{\sqrt{N} \omega}, \hat{a}_{j}^{\dagger}(t) \hat{a}_{k}(t) \phi_{\sqrt{N} \omega}\right\rangle
$$

could be considered in the same way, and the asymptotics as $N \rightarrow+\infty$ recovers, in $L^{p}\left(\mu_{N}\right)$-norm, the quantity

$$
\bar{\Phi}_{t}^{(j)} \Phi_{t}^{(k)}
$$

related to the components $j$ and $k$ of the DNLS flow at time $t$.

Finally, for what concerns the problem of reduced density matrices (see for example $[35,36]$ ), we recall the setting of the operator $\Gamma_{\Psi}: L^{2} \rightarrow L^{2}$ (see Sects. 10.2.1 and 10.2.2 of [21])

$$
\Gamma_{\Psi} \varphi:=\frac{1}{N} \sum_{j, k \geq 0}\left\langle\Psi, b^{*}\left(u_{j}\right) b\left(u_{k}\right) \Psi\right\rangle\left\langle u_{j}, \varphi\right\rangle u_{k}, \quad \varphi \in L^{2}(\mathbb{R} ; \mathbb{C}),
$$

where $\Psi=\Psi^{(N)}$ is fixed in the $N$-particle sector $\mathcal{F}^{(N)} \simeq L_{s}^{2}\left(\mathbb{R}^{N} ; \mathbb{C}\right)$ of the Fock space, and where $b^{*}\left(u_{j}\right)$ and $b\left(u_{k}\right)$ are the bosonic creation and annihilation operators on the Fock space, and associated with a fixed orthonormal set $\left\{u_{j}\right\}_{j \in \mathbb{N}}$ of $L^{2}(\mathbb{R} ; \mathbb{C})$. A preliminary treatment of the link between $\Gamma_{\Psi}$ with the contents of our paper is reported in the $\mathrm{PhD}$ Thesis of one of the authors [34].

\subsection{Fock-Bargmann Space and Wick Quantization}

In this subsection, we provide an overview of Fock-Bargmann space and the Wick quantization, recalling the basic notions we need in the framework of this paper. Here, we mainly follow the notations of Sect. 5.2 in [15], but we also address the reader to Sects. $1.6-2.7$ of $[23]$ and Sect. 2.6 in $[21]$. Let $\overline{\mathcal{A}}\left(\mathbb{C}^{n}\right)$ be the set of the anti-analytic functions $f: \mathbb{C}^{n} \rightarrow \mathbb{C}$. The Fock-Bargmann space is defined as

$$
\mathcal{F}_{B}\left(\mathbb{C}^{n}\right):=\left\{\left.f \in \overline{\mathcal{A}}\left(\mathbb{C}^{n}\right)\left|\int\right| f(\bar{z})\right|^{2} e^{-|z|^{2}} d z \wedge d \bar{z}<+\infty\right\}
$$

with a scalar product

$$
\langle f, g\rangle:=\int f^{\star}(\bar{z}) g(\bar{z}) e^{-|z|^{2}} d z \wedge d \bar{z}
$$




$$
=\frac{1}{\pi^{n}} \int_{\mathbb{R}^{2 n}} f^{\star}(x-i y) g(x+i y) e^{-\left(|x|^{2}+|y|^{2}\right)} d x d y,
$$

here $z:=x+i y$ and $d z \wedge d \bar{z}:=\pi^{-n} d x d y$, thus the integral can be written over $\mathbb{R}^{2 n}$. In this paper, we consider $n=L$ where $L$ is a parameter of the BoseHubbard model. The creation and annihilation operators are defined as:

$$
\hat{b}_{k}(f)(\bar{z}):=\frac{\partial f(\bar{z})}{\partial \bar{z}_{k}} ; \quad \hat{b}_{k}^{\dagger}(f)(\bar{z}):=\bar{z}_{k} f(\bar{z}) .
$$

Notice that $\hat{b}_{k}^{\dagger}, \hat{b}_{k}$ are well defined on $\mathcal{F}_{B}\left(\mathbb{C}^{n}\right)$ and $\left[\hat{b}_{k}, \hat{b}_{\mu}^{\dagger}\right]=\delta_{k \mu}$ Id. Coherent states are represented, with its normalization $e^{-\frac{1}{2}|\omega|^{2}}$, as

$$
\phi_{\omega}(\bar{z}):=e^{\omega \cdot \bar{z}-\frac{1}{2} \omega \cdot \bar{\omega}} .
$$

For a given operator $\widehat{A}: \mathcal{F}_{B}\left(\mathbb{C}^{n}\right) \rightarrow \mathcal{F}_{B}\left(\mathbb{C}^{n}\right)$, its Wick symbol is defined by

$$
\sigma(\widehat{A})(\bar{\omega}, \omega):=\left\langle\phi_{\omega}, \widehat{A} \phi_{\omega}\right\rangle
$$

whereas outside the diagonal $(\bar{\omega}, \omega)$ the Wick symbol reads

$$
\sigma(\widehat{A})(\bar{z}, \omega):=\frac{\left\langle\phi_{z}, \widehat{A} \phi_{\omega}\right\rangle}{\left\langle\phi_{z}, \phi_{\omega}\right\rangle} .
$$

The Wick quantization of an entire function $\sigma: \mathbb{C}^{n} \times \mathbb{C}^{n} \rightarrow \mathbb{C}$ is given as

$$
\mathrm{Op}_{\mathrm{W}}(\sigma)(f)(\bar{z}):=\int \sigma(\bar{z}, \omega) f(\bar{\omega}) e^{-|\omega|^{2}+\omega \cdot \bar{z}} d \omega \wedge d \bar{\omega}, \quad f \in \mathcal{F}_{B}\left(\mathbb{C}^{n}\right) .
$$

In view of these settings, we have $\widehat{A}=\mathrm{Op}_{\mathrm{W}}(\sigma(\widehat{A}))$, and we call this a Wick operator.

To be more precise about the set of these operators, suppose that $\widehat{A}$ (possibly unbounded) is defined on $\mathcal{F}_{B}\left(\mathbb{C}^{n}\right)$ together with its adjoint $\widehat{A}^{\dagger}$, and assume that for all $\omega \in \mathbb{C}^{L}, \phi_{\omega}$ belongs to the domains of $\widehat{A}$ and $\widehat{A}^{\dagger}$. Then, $\omega \mapsto \sigma(\widehat{A})(\bar{\omega}, \omega)$ is a smooth function on $\mathbb{C}^{n}$ and moreover $\sigma(\widehat{A})(\bar{\omega}, \omega)$ is the restriction on the diagonal of $\sigma(\widehat{A})(\bar{z}, \omega)$ as in (137), which is furthermore an entire function (see [23] - pg. 139). As shown in Proposition 1.69 of [23], any entire function $K(\bar{z}, \omega)$ is uniquely determined by its restriction to $\{\bar{z}=\bar{\omega}\}$.

Thanks to these observations, $\widehat{A}=\mathrm{Op}_{\mathrm{W}}(\sigma)$ is uniquely related to the symbol on the diagonal, and for this reason frequently in the literature one refers to $\widehat{A}$ as the Wick quantization of (136).

We also stress that if the starting point is the definition (138) one must prove, for a given entire function $\sigma$, that $\mathrm{Op}_{\mathrm{W}}(\sigma)$ is well defined on $\mathcal{F}_{B}\left(\mathbb{C}^{n}\right)$ is the sense we have just described.

A simple computation shows that

$$
\sigma\left(\hat{b}_{k}\right)=\omega_{k}, \quad \sigma\left(\hat{b}_{k}^{\dagger}\right)=\bar{\omega}_{k}, \quad \sigma\left(\left(\hat{b}_{k}^{\dagger}\right)^{\alpha}\left(\hat{b}_{\mu}\right)^{\beta}\right)=\bar{\omega}_{k}^{\alpha} \omega_{\mu}^{\beta} .
$$


These equalities directly allow to write the Wick symbol of the Bose-Hubbard operator $\widehat{H}$ in $(2)$

$$
\left\langle\phi_{\omega}, \widehat{H} \phi_{\omega}\right\rangle=\sum_{1 \leq j \leq n}\left[E\left|\omega_{j}\right|^{2}+J\left(\bar{\omega}_{j+1} \omega_{j}+\bar{\omega}_{j} \omega_{j+1}\right)+\frac{U}{2 N}\left|\omega_{j}\right|^{4}\right]
$$

and the rescaled Wick symbol

$$
\left\langle\phi_{\sqrt{N} \omega}, \widehat{H} \phi_{\sqrt{N} \omega}\right\rangle=N \sum_{1 \leq j \leq n}\left[E\left|\omega_{j}\right|^{2}+J\left(\bar{\omega}_{j+1} \omega_{j}+\bar{\omega}_{j} \omega_{j+1}\right)+\frac{U}{2}\left|\omega_{j}\right|^{4}\right] .
$$

We also recall that any bounded operator on $\mathcal{F}_{B}\left(\mathbb{C}^{n}\right)$ is a well-defined Wick operator. Furthermore, a large class of Weyl operators (see Sect. 2.1 in [23]) can be rewritten as a Wick operator by the following link of symbols $\sigma_{\text {Wick }}=$ $e^{\Delta / 2} \sigma_{\text {Weyl }}$, namely (for $\hbar=1$ )

$$
\sigma_{\mathrm{Wick}}(\bar{\omega}, \omega)=2^{n} \int e^{-2(z-\omega)(\bar{z}-\bar{\omega})} \sigma_{\text {Weyl }}\left(\frac{z+\bar{z}}{\sqrt{2}}, \frac{z-\bar{z}}{\sqrt{2}}\right) d z \wedge d \bar{z},
$$

see also Proposition 2.97 in [23] for the link with standard quantization, and more detailed setting for the allowed symbols.

The set of Wick operators is closed under composition, and the Wick-^ product is defined as the symbol of the composition of two operators,

$$
\left(\sigma_{1} \star_{\mathrm{Wick}} \sigma_{2}\right)(\bar{\omega}, \omega):=\left\langle\phi_{\omega}, \mathrm{Op}_{\mathrm{W}}\left(\sigma_{1}\right) \circ \mathrm{Op}_{\mathrm{W}}\left(\sigma_{2}\right) \phi_{\omega}\right\rangle .
$$

It can be shown (see [15]) the following asymptotics (in multi-index notation)

$$
\begin{aligned}
\sigma_{1} \star_{\text {Wick }} \sigma_{2} & \simeq \sum_{r=0}^{\infty} \frac{1}{r !} \frac{\partial^{r} \sigma_{1}}{\partial \omega^{r}} \frac{\partial^{r} \sigma_{2}}{\partial \bar{\omega}^{r}} \\
& \simeq \sum_{r=0}^{\infty} \frac{1}{r !} \sum_{i_{1}, i_{2}, \ldots i_{r}=1}^{n} \frac{\partial^{r} \sigma_{1}}{\partial \omega_{i_{1}} \partial \omega_{i_{2}} \ldots \partial \omega_{i_{r}}} \frac{\partial^{r} \sigma_{2}}{\partial \bar{\omega}_{i_{1}} \partial \bar{\omega}_{i_{2}} \ldots \partial \bar{\omega}_{i_{r}}},
\end{aligned}
$$

where $\partial \omega^{r}:=\partial \omega_{i_{1}} \partial \omega_{i_{2}} \ldots \partial \omega_{i_{r}}$.

About the convergence of the right-hand side, we address the reader to [9]. We stress that in the asymptotics (144) the semiclassical parameter is $\hbar=1$ and the absence of the factor $2^{r}$ used in [9] is a consequence of the setting of the scalar product in (133). reads

In the case of the rescaled symbols $\sigma(\sqrt{N} \bar{\omega}, \sqrt{N} \omega)$, the Wick-star product

$$
\sigma_{1} \star_{\text {Wick }} \sigma_{2} \simeq \sum_{r=0}^{\infty} \frac{\hbar^{r}}{r !} \frac{\partial^{r} \sigma_{1}}{\partial \omega^{r}} \frac{\partial^{r} \sigma_{2}}{\partial \bar{\omega}^{r}}, \quad \hbar=\frac{1}{N} .
$$

One can also introduce the $\hbar$-Wick quantization

$$
\mathrm{Op}_{\mathrm{W}}^{\hbar}(\sigma)(f)(\bar{z}):=\hbar^{-n} \int \sigma(\bar{z}, \omega) f(\bar{\omega}) e^{-\frac{1}{\hbar}\left(|\omega|^{2}-\omega \cdot \bar{z}\right)} d \omega \wedge d \bar{\omega}
$$


for which we have the Wick-^ product of symbols in (145), defined on the FockBargmann space with the scalar product $\langle\psi, \varphi\rangle:=\hbar^{-n} \int \psi^{\star}(\bar{z}) \varphi(\bar{z}) e^{-\frac{1}{\hbar}|z|^{2}} d z \wedge$ $d \bar{z}$.

The Wick bracket is defined as the symbol of the commutator

$$
\left\{\sigma_{1}, \sigma_{2}\right\}_{\text {Wick }}:=\sigma_{1} \star_{\text {Wick }} \sigma_{2}-\sigma_{2} \star_{\text {Wick }} \sigma_{1} \text {. }
$$

For trace class Wick operators, a very useful formula gives

$$
\operatorname{Tr}\left(\mathrm{Op}_{\mathrm{W}}(\sigma)\right)=\int \sigma(\bar{\omega}, \omega) d \omega \wedge d \bar{\omega} .
$$

Remark 4. Let $U(t):=e^{-i \widehat{H} t}$ and $\hat{b}_{k}(t):=U^{\dagger}(t) \hat{b}_{k} U(t)$. Recalling Remark 2, we have that $\hat{b}_{k}(t)$ is a Wick operator for any $t \geq 0$. We denote now its symbol by $\sigma_{k}(t, \bar{\omega}, \omega)$. Moreover, it is easy to see that $\rho_{k}(t, \bar{\omega}, \omega):=\left\langle\phi_{\sqrt{N} \omega}, \hat{a}_{k}(t) \phi_{\sqrt{N} \omega}\right\rangle$ fulfills

$$
\rho_{k}(t, \bar{\omega}, \omega)=\frac{1}{\sqrt{N}} \sigma_{k}(t, \sqrt{N} \bar{\omega}, \sqrt{N} \omega)
$$

hence the operator $\mathrm{Op}_{\mathrm{W}}\left(\rho_{k}\right)$ is well defined in the Wick quantization.

\subsection{Remarks on Phase Space Analysis}

Let $\Psi(t)$ be the solution of the quantum dynamics for some fixed initial data $\Psi(0) \in \mathcal{F}_{B}\left(\mathbb{C}^{L}\right)$, for example a normalized function in the $N$-sector of $\mathcal{F}_{B}\left(\mathbb{C}^{L}\right)$ whence representing $N$-particle states (see [23], pg. 48) and $\widehat{\Pi}_{\Psi(t)}$ the related projection operator. Let us consider the related Wick symbol $\sigma_{0}(\bar{\omega}, \omega):=\sigma_{W}\left(\widehat{\Pi}_{\Psi(0)}\right)(\bar{\omega}, \omega)=\left|\left\langle\phi_{\omega}, \Psi(0)\right\rangle\right|^{2}$. A way to study the semiclassical localization of the operator

$$
\widehat{B}(t):=\widehat{\Pi}_{\Psi(t)}-\mathrm{Op}_{\mathrm{W}}\left(\sigma_{0} \circ \Phi_{t}\right)
$$

as the parameter $h=1 / N \rightarrow 0$ is to describe the essential support (see Theorem 8.16 in [41]). In order to do this, one has to study the semiclassical Wave Front set, also called Frequency Set (see for example [31], or [18] for the periodic setting which is the one of the current paper)

$$
W F_{s}\left(\widehat{B}(t) \psi_{\alpha}\right)
$$

where $\psi_{\alpha}$ belongs to an orthonormal set, given for example by eigenfunctions labeled by the multi-index $\alpha \in \mathbb{N}^{L}$ of the number operator $\widehat{N}:=\sum_{k} \widehat{b}^{\dagger} \widehat{b}_{k}$. The phase space localization of this set can be described by the use of the coherent states $\phi_{\sqrt{N} \omega}$ and study of the FBI transform $\left\langle\phi_{\sqrt{N} \omega}, \widehat{B}(t) \psi_{\alpha}\right\rangle$ (see Sect. 3.6point 3 of [31]) which reads (thanks to coherent states decomposition)

$$
\begin{aligned}
\left\langle\phi_{\sqrt{N} \omega}, \widehat{B}(t) \psi_{\alpha}\right\rangle=\int\left\langle\phi_{s}, \psi_{\alpha}\right\rangle\left\langle\phi_{\sqrt{N} \omega}, \widehat{B}(t) \phi_{s}\right\rangle d s \wedge d \bar{s} \\
=\int e^{-\frac{1}{2}|s|^{2}} \frac{s^{\alpha}}{\sqrt{\alpha !}}\left\langle\phi_{\sqrt{N} \omega}, \widehat{B}(t) \phi_{s}\right\rangle d s \wedge d \bar{s} \\
=\int N^{L} e^{-\frac{1}{2} N|v|^{2}} \frac{(\sqrt{N} v)^{\alpha}}{\sqrt{\alpha !}}\left\langle\phi_{\sqrt{N} \omega}, \widehat{B}(t) \phi_{\sqrt{N} v}\right\rangle d v \wedge d \bar{v} .
\end{aligned}
$$


Whence,

$$
\left|\left\langle\phi_{\sqrt{N} \omega}, \widehat{B}(t) \psi_{\alpha}\right\rangle\right| \leq \int N^{L} e^{-\frac{1}{2} N|v|^{2}} \frac{(\sqrt{N}|v|)^{|\alpha|}}{\sqrt{\alpha !}}\left|\left\langle\phi_{\sqrt{N} \omega}, \widehat{B}(t) \phi_{\sqrt{N} v}\right\rangle\right| d v \wedge d \bar{v} .
$$

Notice that these are Gaussian-type integrals. Our estimate in Proposition 3 can be obtained in the same way for $\left|\left\langle\phi_{\sqrt{N} \omega}, \widehat{B}(t) \phi_{\sqrt{N} v}\right\rangle\right|$ (i.e., also outside the diagonal $\omega=v$ ), where instead of the evolved annihilation operator $\widehat{a}_{k}(t)$ we have $\widehat{\Pi}_{\Psi(t)}$. Thus, our $L^{2}$-estimates in Theorem 1 with respect to Gaussian measures can be easily adapted to get a semiclassical estimate for the integral (152) with a more general invariant measure. To conclude, we stress that higher-order corrections of (150) implies better localization estimates for the function $\omega \mapsto\left\langle\phi_{\sqrt{N} \omega}, \widehat{B}(t) \psi_{\alpha}\right\rangle$ in (152). This can be done in the spirit of Egorov Theorem (see $[8,16]$ ) by iterating the semigroup identity (70) working on the time evolved Wick symbols governed by Eq. (8).

\section{References}

[1] Ablowitz, M.J., Curtis, C.W., Zhu, Y.: On tight-binding approximations in optical lattices. Stud. Appl. Math. 129, 362 (2012)

[2] Ablowitz, M.J., Prinari, B., Trubatch, A.D.: Discrete and Continuous Nonlinear Schrödinger Systems. Cambridge University Press, Cambridge (2003)

[3] Abramowitz, M., Stegun, I.A.: Handbook of Mathematical Functions. Dover, New York (1972)

[4] Adami, R., Golse, F., Teta, A.: Rigorous derivation of the cubic NLS in dimension one. J. Stat. Phys. 127(6), 1193-1220 (2007)

[5] Ammari, Z., Nier, F.: mean field limit for bosons and infinite dimensional phasespace analysis. Ann. Henri Poincaré 9, 1503-1574 (2008)

[6] Anapolitanos, I., Hott, M.: A simple proof of convergence to the Hartree dynamics in Sobolev trace norms. J. Math. Phys. 57, 122108 (2016)

[7] Bach, V., Breteaux, S., Chen, T., Fröhlich, J., Sigal, I. M.: The time-dependent Hartree-Fock-Bogoliubov equations for Bosons. arXiv:1602.05171

[8] Bambusi, D., Graffi, S., Paul, T.: Long time semiclassical approximation of quantum flows: a proof of the Ehrenfest time. Asymptot. Anal. 21,(1999)

[9] Beiser, S., Römer, H., Waldmann, S.: Convergence of the Wick product. Commun. Math. Phys. 272, 25-52 (2007)

[10] Benedikter, N., Sok, J., Solovej, J.P.: The Dirac-Frenkel principle for reduced density matrices, and the Bogoliubov-de Gennes equations. Ann. Henri Poincaré 19, 1167-1214 (2018)

[11] Bobmann, L., Pavlović, N., Pickl, P., Soffer, A.: Higher order corrections to the mean-field description of the dynamics of interacting bosons. J. Stat. Phys. 178, 1362-1396 (2020)

[12] Bloch, I., Dalibard, J., Zwerger, W.: Many-body physics with ultracold gases. Rev. Mod. Phys. 80, 885-964 (2008)

[13] Boccato, C., Cenatiempo, S., Schlein, B.: Quantum many-body fluctuations around nonlinear Schrödinger dynamics. Ann. Henri Poincaré 18, 113-191 (2017) 
[14] Benedikter, N., de Oliveira, G., Schlein, B.: Quantitative derivation of the GrossPitaevskii equation. Commun. Pure Appl. Math. 68(8) (2015)

[15] Berezin, F.A., Shubin, M.: The Schrödinger Equation. Mathematics and its Applications, Springer, Berlin (1991)

[16] Bouzouina, A., Robert, D.: Uniform semi-classical estimates for the propagation of quantum observables. Duke Math. J. 111(2) (2002)

[17] Brennecke, C., Nam, P.T., Napiórkowski, M., Schlein, B.: Fluctuations of Nparticle quantum dynamics around the nonlinear Schrödinger equation. Annales de Institut Henri Poincaré C, Analyse non linéaire 36(5), 1201-1235 (2019)

[18] Cardin, F., Zanelli, L.: The geometry of the semiclassical wave front set for Schrödinger eigenfunctions on the torus. Math. Phys. Anal. Geom. 20, 10 (2017)

[19] Cazalilla, M.A., Citro, R., Giamarchi, T., Orignac, E., Rigol, M.: One dimensional Bosons: from condensed matter systems to ultracold gases. Rev. Mod. Phys. 83, 1405 (2011)

[20] Chen, X., Holmer, J.: Focusing quantum many-body dynamics: the rigorous derivation of the $1 \mathrm{~d}$ focusing cubic nonlinear Schrödinger equation. Arch. Ration. Mech. Anal. 221(2), 631-676 (2016)

[21] Combescure, M., Robert, D.: Coherent States and Applications in Mathematical Physics. Springer, Berlin (2012)

[22] Eilbeck, J.C., Johansson, M.: The discrete nonlinear Schrödinger equation -20 years on. Proc. of the third Conference: Localization and Energy Transfer in Nonlinear Systems, pp. 44-67, World Scientific (2003)

[23] Folland, G.: Harmonic Analysis in Phase Space. (AM-122), Volume 122. Princeton University Press, Annals of Mathematics Studies (1989)

[24] Fröhlich, J., Graffi, S., Schwarz, S.: Mean field and classical limit of many-body Schrödinger dynamics for bosons. Commun. Math. Phys. 271, 681-697 (2007)

[25] Fröhlich, J., Knowles, A., Schlein, B., Sohinger, V.: Gibbs measures of nonlinear Schrödinger equations as limits of many-body quantum states in dimensions $d \leq 3$. Commun. Math. Phys. 356, 883-980 (2017)

[26] Fröhlich, J., Knowles, A., Schwarz, S.: On the mean-field limit of Bosons with coulomb two-body interaction. Commun. Math. Phys. 288, 1023-1059 (2009)

[27] Ginibre, J., Velo, G.: The classical field limit of scattering theory for nonrelativistic many-boson systems. I. Commun. Math. Phys. 66, 37-76 (1979)

[28] Jeblick, M., Leopold, N., Pickl, P.: Derivation of the time dependent GrossPitaevskii equation in two dimensions. Commun. Math. Phys. 372(1), 1-69 (2019)

[29] Hepp, K.: The classical limit for quantum mechanical correlation functions. Commun. Math. Phys. 35, 265-277 (1974)

[30] Lahini, Y., Steinbrecher, G. R., Bookatz, A. D.: Dirk Englund: Quantum logic using correlated one-dimensional quantum walks. npj Quant. Inf. 4(2) (2018)

[31] Martinez, A.: An Introduction to Semiclassical and Microlocal Analysis. Springer, Berlin (2002)

[32] Michelangeli, A., Olgiati, A.: Mean-field quantum dynamics for a mixture of Bose-Einstein condensates. Anal. Math. Phys. 7(4), 377-416 (2017) 
[33] Pelinovsky, D.E., Schneider, G.: Bounds on the tight-binding approximation for the Gross-Pitaevskii equation with a periodic potential. J. Diff. Equ. 248, 837 (2010)

[34] Picari, E.: Coherent Quantum Dynamics of Bosons in Measure. University of Padova, Italy (2021). (PhD Thesis in Mathematics)

[35] Pickl, P.: A simple derivation of mean field limits for quantum systems. Lett. Math. Phys. 97, 151-164 (2011)

[36] Rodnianski, I., Schlein, B.: Quantum fluctuations and rate of convergence towards mean field dynamics. Commun. Math. Phys. 291, 31-61 (2009)

[37] Sacchetti, A.: Derivation of the tight-binding approximation for time-dependent nonlinear Schrödinger equations. Ann. Henri Poincaré 21, 627-648 (2020)

[38] Seiringer, R.: Bose gases, Bose-Einstein condensation, and the Bogoliubov approximation. J. Math. Phys. 55, 075209 (2014)

[39] Spohn, H.: Kinetic equations from Hamiltonian dynamics: Markovian limits. Rev. Mod. Phys. 52(3), 569-615 (1980)

[40] Trombettoni, A., Smerzi A.: Discrete solitons and breathers with dilute BoseEinstein condensates. Phys. Rev. Lett. 86(11) (2001)

[41] Zworski M.: AMS: Semiclassical Analysis, (Graduate Studies in Mathematics) (2012)

E. Picari, A. Ponno and L. Zanelli

Department of Mathematics

"Tullio Levi-Civita" University of Padova

Padua

Italy

e-mail: enrico.dsf@gmail.com;

ponno@math. unipd.it;

lzanelli@math.unipd.it

Communicated by Vieri Mastropietro.

Received: October 8, 2020.

Accepted: September 16, 2021. 